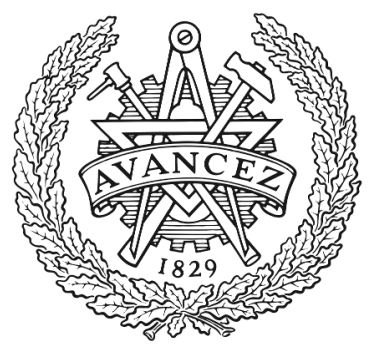

CHALMERS

UNIVERSITY OF TECHNOLOGY

\title{
Iterative time-reversal for multi-frequency hyperthermia
}

Downloaded from: https://research.chalmers.se, 2023-04-26 10:43 UTC

Citation for the original published paper (version of record):

Zanoli, M., Dobsicek Trefna, H. (2021). Iterative time-reversal for multi-frequency hyperthermia.

Physics in Medicine and Biology, 66(4). http://dx.doi.org/10.1088/1361-6560/abd41a

N.B. When citing this work, cite the original published paper. 


\title{
Physics in Medicine \& Biology
}

\section{PAPER}

CrossMark

\section{Iterative time-reversal for multi-frequency hyperthermia}

\section{OPEN ACCESS}

\section{RECEIVED}

12 October 2020

REVISED

7 December 2020

ACCEPTED FOR PUBLICATION

16 December 2020

PUBLISHED

10 February 2021

Original content from thi work may be used under the terms of the Creative

Commons Attribution 4.0 licence.

Any further distribution of this work must maintain

attribution to the author(s) and the title of the work, journal citation and DOI.

\author{
Massimiliano Zanoli $(1)$ and Hana Dobšíček Trefná $(1)$ \\ Dept. Electrical Engineering, Chalmers University of Technology, Gothenburg, SE, Sweden \\ E-mail: hanatre@chalmers.se \\ Keywords: microwave hyperthermia, time reversal, hot-spot, multi-frequency
}

\begin{abstract}
Time-reversal (TR) is a known wideband array beam-forming technique that has been suggested as a treatment planning alternative in deep microwave hyperthermia for cancer treatment. While the aim in classic TR is to focus the energy at a specific point within the target, no assumptions are made on secondary lobes that might arise in the healthy tissues. These secondary lobes, together with tissue heterogeneity, may result in hot-spots (HSs), which are known to limit the efficiency of the thermal dose delivery to the tumor. This paper proposes a novel wideband TR focusing method that iteratively shifts the focus away from HSs and towards cold-spots from an initial TR solution, a procedure that improves tumor coverage and reduces HSs. We verify this method on two different applicator topologies and several target volume configurations. The algorithm is deterministic and runs within seconds, enabling its use for real-time applications. At the same time, it yields results comparable to those obtained with global stochastic optimizers such as Particle Swarm.
\end{abstract}

\section{Introduction}

In deep microwave hyperthermia (MW-HT) for cancer treatment, a conformal array of antennas, called applicator, is used to non-invasively and selectively increase the temperature of a deep-seated tumor up to $40{ }^{\circ} \mathrm{C}-44^{\circ} \mathrm{C}$ for approximately one hour (Elming et al 2019 , Paulides et al 2020). Randomized clinical trials have demonstrated the efficacy of this technique in enhancing the therapeutic effects of chemo- and radio-therapy (Cihoric et al 2015, Datta et al 2015, 2016). The challenge in MW-HT is to reach deep-seated targets with adequate power deposition while keeping the nearby healthy tissues (HSs) below a safety temperature threshold. These HSs lie in the path between the antenna and the target, and they tend to absorb energy from the traveling wave, as a consequence of their relatively high conductivity at RF frequencies. The power loss in these tissues can cause hot-spots (HSs), which effectively limit the maximum temperature achievable in the target. This can prevent the treatment from reaching high therapeutic thermal doses (Paulides et al 2010, Kok et al 2017). Superficial HSs within depths of one to two centimeters from the skin can be suppressed by applying a water bolus circulated with cool water. Other HSs might arise deeper in the body due to the anatomical heterogeneity and interfaces between tissues with different dielectric properties, together with the imperfect interference pattern generated by the phased array. In such cases, the pattern has to be improved by means of amplitude and phase steering of the applicator array, with parameters obtained by a hyperthermic treatment planning (HTP) step (Gavazzi et al 2020).

Several methods for determining the optimal amplitudes and phases for an applicator array yielding satisfying target coverage and limited HS temperatures have been developed in the past and are still subject of ongoing research (Paulides et al 2013, Kok et al 2015). These methods can be classified into two main categories: specific absorption rate (SAR) based and temperature ( $T$ ) based. SAR based techniques rely on the assumption that the SAR distribution is predictive of the temperature distribution in the patient (Canters et al 2009). However, since the thermal response of the body can be highly nonlinear, SAR-based optimizers can yield suboptimal, while still clinically relevant, HTP parameters. T-based optimizers, on the other hand, have evolved to include complex nonlinear aspects such as discrete vasculature and systemic response under thermal stress (Kok et al 2013). Ideally, a full implementation of T-based HTP in the clinical routine is desirable, since temperature is 
the objective dose. In current practice, however, the theoretical benefits of $T$-based HTP are somewhat diminished due to the lack of accurate estimations of the thermal tissue properties (De Greef et al 2010, Canters et al 2012, 2013). Such thermal properties, and blood perfusion in particular, exhibit large variations across patients and even at individual level in different treatment sessions. Therefore, both SAR-based and T-based approaches are being clinically applied as both require adjustments during treatment in response to hot spots (Rijnen et al 2013, Kok et al 2017). Considering the current equivalence of these two approaches, in the present work we consider only SAR based HTP techniques.

Among them, the eigenvalue (EV) and, more recently, the particle swarm (PS) algorithms have become the most established in the literature (Paulides et al 2013, Kok et al 2015). The EV method maximizes the ratio between the average SAR in the target and in the remaining tissues (Böhm et al 1993, Bardati et al 1995). When the resulting temperature distribution is showing overheated healthy regions, a weighing factor can be introduced to iteratively reduce the power deposition at those locations (Köhler et al 2001). EV is a direct method that provides a fast and deterministic solution, but its main limitation lies in the form of the cost function, which has to be a quadratic ratio between polynomials. Quadratic cost function predictions have been shown to correlate poorly with the SAR or temperature rise in the tumor target volume during clinical treatment (Canters et al 2009). For this reason, more clinically relevant and temperature-predicting indicators such as the hotspot-to-target quotient (HTQ) have been proposed (Canters et al 2010). Since the evaluation of the HTQ involves at least one nonlinear step with respect to the complex array steering parameters, EV cannot be directly used to determine the HTQ-optimal solution. A second limitation of EV occurs with multi-frequency optimization: the generalized EV form can not be defined for multiple simultaneous operating frequencies in a conjoint manner (Trefná et al 2017, Zanoli and Trefná 2020).

There is mounting evidence that the development of UWB applicators capable of operating at different frequencies will lead to improved target coverage and HS suppression (Converse et al 2006, Trefná et al 2010a, Bellizzi et al 2017, Kuehne et al 2020). An UWB system can exploit the complementary interference patterns generated by different frequencies to increase the net average power delivered to the tumor while reducing the absorption in HSs. The benefit of UWB is even more pronounced for the treatment of tumors in challenging locations, such as the brain (Winter et al 2015, Takook et al 2018). The brain region is in fact characterized by a combination of anatomical and physiological factors that require an extra degree of accuracy in the formation of the heating pattern. The cerebral tissue is more sensitive to deviations in temperature than other tissues (Yarmolenko et al 2011), and the presence of cerebrospinal fluid causes strong HSs to appear in the immediate vicinity of the brain (Schooneveldt et al 2019). The single-frequency constraint of EV might therefore represent an insurmountable limitation for multi-frequency HTP.

PS is another type of HTP optimizer used in clinical practice (Paulides et al 2013). If the PS optimization is configured to minimize the HTQ, the resulting steering settings can exhibit significant differences from those given by EV. In particular, the resulting raw power deposition ratio might be worse than the one obtained via EV, but the SAR distribution is often better in terms of more relevant clinical parameters: target coverage and heating homogeneity. Furthermore, the PS method can easily be extended for use with multiple operating frequencies. The drawback with PS, being a stochastic algorithm, is its long execution time needed to consistently converge to the same global optimum between different executions. A typical run with 100 iterations, as usually required for reasonable accuracy, can take half an hour or more to complete, depending on the model resolution.

Time reversal (TR) focusing is a fast, intrinsically wide-band and deterministic method that has been proposed and validated as an alternative microwave HTP technique (Guo et al 2005, Trefná et al 2010b). TR processing is a well characterized inverse filter initially developed for focusing ultrasound pulses generated by transducer arrays inside a biological target (Thomas and Fink 1996, Fink et al 2004), and subsequently extended for use with electromagnetic antenna arrays (Lerosey et al 2004). It exploits the time and space reciprocity of the wave equation to determine the optimal phase and amplitude settings of an array of radiators that will cause the highest constructive interference to occur at the desired location. In its basic implementation, with a single virtual source placed at the center of the tumor, TR already exhibits improved target coverage and HTQ than EV (Trefná et al 2010a, 2010b, Takook et al 2015), though not comparable to PS optimization. Further improvements for HS-management in TR have been proposed for high-intensity focused ultrasounds (HIFU), based on iterative methods (Leduc et al 2012). In general, the major hindrance for the clinical introduction of TR-based HTP is its limited ability to suppress HSs. In this work, we present a novel deterministic HS suppression algorithm for TR-based HTP that yields results comparable to those obtained via global PS optimization, while being significantly faster. The rest of the paper is organized as follows: we first introduce the iterative procedure in detail, to later benchmark its performances numerically for two different applicator array topologies: a collar for tumors in the neck and a helmet for tumors in the brain. For the neck model, we further consider different target locations and sizes, to assess the algorithm's robustness to different scenarios. For each test case, HTP quality and performance indicators are computed and compared against those obtained with EV, PS and classic TR. 


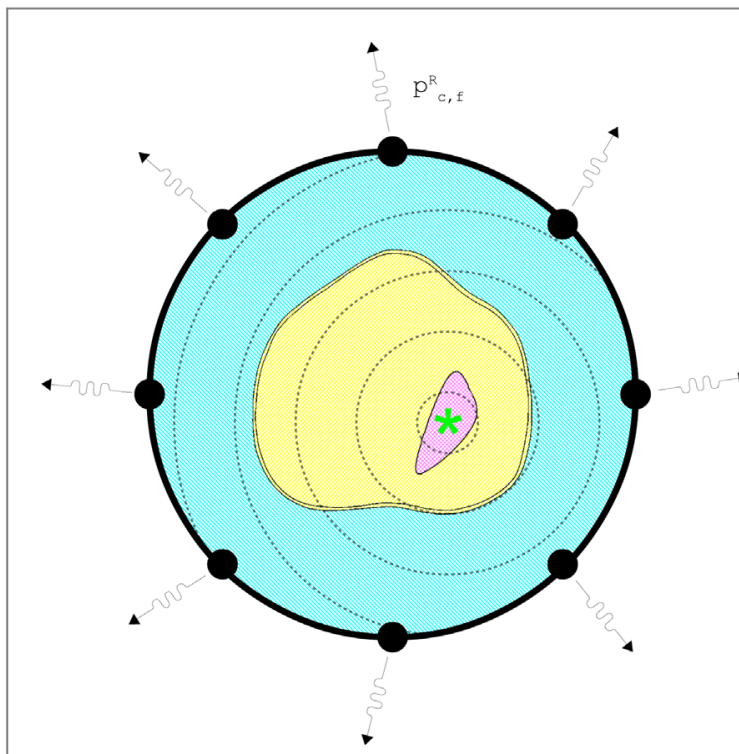

(a) Recording phase.

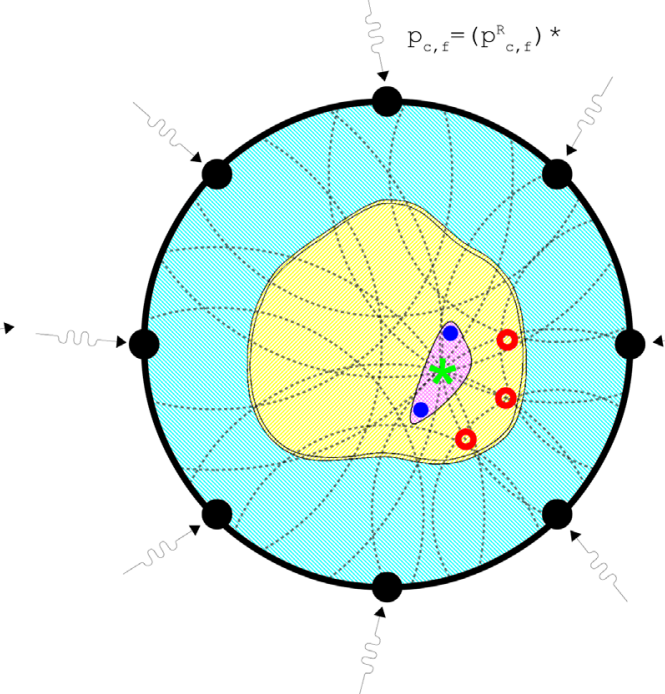

(b) Steering phase.

Figure 1. Conceptual scheme for time-reversal treatment planning, example in the neck region. In the recording phase (a) a virtual source (green asterisk) is placed at the desired focal spot inside the tumor. The source is then excited and the phasors $p_{c, f}^{R}$ are determined. In the steering phase (b) the conjugate phasors are used as steering parameters to obtain a focal spot where the virtual source was located. Due to imperfect interference, hot-spots (red circles) arise in the HSs and cold-spot (blue dots) can be identified in the target volume.

\section{Theory}

\subsection{Classic TR focusing}

TR focusing is achieved by placing a so-called virtual source at the desired focal spot and by recording the impulse response at the antenna locations (figure 1(a)). The recorded signals are then back-propagated simultaneously by all the antennas, generating an interference pattern that exhibits full phase coherence at the original source location (figure 1(b)). Together with the desired peak, however, secondary lobes appear due to both the limited aperture of the array and the anatomy of the patient. In the frequency domain, the TR operator corresponds to a complex conjugation $\left(^{*}\right)$ :

$$
p_{c}(f)=\left[p_{c}^{R}(f)\right]^{*}
$$

where $p_{c}^{R}$ is the complex phasor recorded by the array channel $c$ at frequency $f$ during source excitation, while $p_{c}$ is the complex steering parameter for the same channel to focus at the source location. Under this perspective, a discrete set of simultaneous operating frequencies can be selected for treatment, rather than wideband pulses. In practice, an applicator can be operated to switch between single frequencies in finely interleaved time slots, if the duration of each slot is small compared to the biological response (Trefná et al 2017). This simplifies the complexity of the hardware needed for feeding the HT applicator (Trefná et al 2012). The rest of the analysis will therefore be carried out assuming that $n_{f}$ simultaneous operating frequencies can be independently controlled in phase and amplitude for each of the $n_{c}$ channels of the array applicator.

TR focusing requires in principle only one EM simulation per virtual source, which makes it attractive in terms of computational resources. Unfortunately, this single simulation is not sufficient to assess the quality of the resulting treatment planning. The HTP quality indicators must in fact be evaluated over the focused SAR distribution (figure 1(b)). Moreover, since the immediate TR solution for HTP might not be optimal or clinically viable, further adjustments might be needed, which would require additional simulations. In view of this, it is more convenient to run a separate simulation for each antenna/channel $c$ in the array, as is the case for EV or PS. The TR solution at any point can then be determined from this set of E-field distributions via a generalized and source-free TR method described later in section 2.2. Denoting with ${ }^{t}$ the transpose operation (without conjugate), the complex E-field distributions and steering parameters relative to all channels at a certain frequency can be joined into column vectors for compactness:

$$
\boldsymbol{p}_{f}=\left[\begin{array}{lllll}
p_{1, f} & p_{2, f} & \cdots & p_{n_{c}, f}
\end{array}\right]^{t} \quad \boldsymbol{E}_{f}=\left[\begin{array}{llll}
E_{1, f} & E_{2, f} & \ldots & E_{n_{c}} f
\end{array}\right]^{t} .
$$




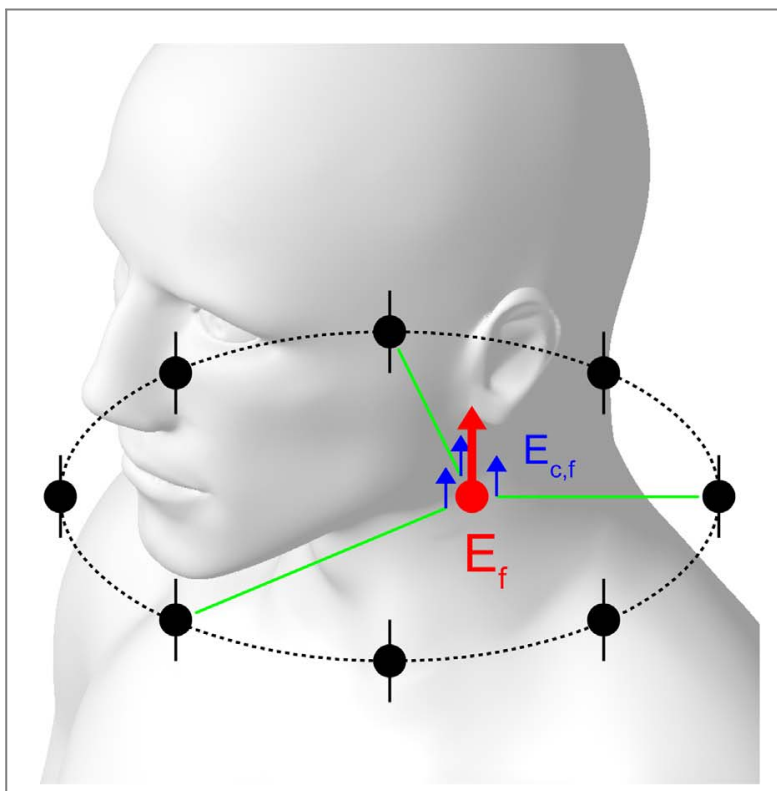

(a) Collar applicator, neck.

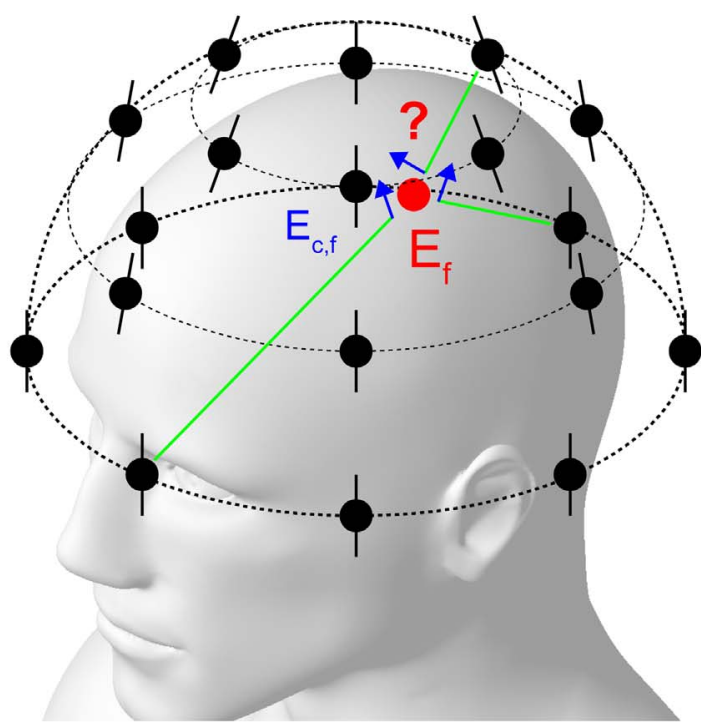

(b) Helmet applicator, brain.

Figure 2. The virtual source polarization problem. In the collar case (a) the main polarization of the fields interfering at the focal spot (red dot) is evident. In the helmet case (b) each antenna contributes to the power loss at the focal spot along a different polarization axis.

\subsection{Challenges for TR focusing in MW-HT}

A number of challenges arise when implementing TR as HTP. First of all, the choice of the source location, or focal spot. Intuitively, the source should be placed at the center of the tumor, but this is often hard to define, especially for irregular shapes. The center of mass of the tumor can be picked as an initial guess, but this choice often leads to inhomogeneous or insufficient heating of deep or peripheral tumor areas. Cold-spots in the target volume should be avoided, as they reduce the treatment's efficacy. Better coverage can be achieved, for example, by translating the focal spot progressively towards the internal, deeper part of the target volume (Takook et al 2017). Second, HSs arising in healthy tissues must be suppressed, to allow a higher deposition in the target. In order to shift the focus away from these locations, the natural approach under the TR perspective would be to place secondary sources, record their impulse response, and subtract these secondary solutions from the primary one. Taken together, these considerations suggest that an initial TR solution with source at the tumor center can be iteratively improved by shifting the set of complex steering parameters away from HSs and towards cold-spots.

A second aspect of TR focusing is the choice of the polarization axis for the virtual source. The polarization problem in vector field focusing has been addressed in several ways in the literature, also recently (Iero et al 2016, Battaglia et al 2020). In the case of a cylindrical applicator, the antennas can be aligned to the symmetry axis (figure 2(a)): the virtual source should then also be aligned with this principal axis. In more complex array topologies such as a semi-spherical helmet, the E-field generated by each antenna can vary significantly in polarization, depending on the location of the focal spot (figure 2(b)). The resulting interference is no longer characterized by a single main polarization, and the concept of virtual source becomes inadequate. This concept should then be dropped in favor of the more generalized concept of complex power contribution at the focal spot. We know that the SAR at a location $\zeta$ resulting from the superposition of all channel fields at a particular frequency $f$ is given by:

$$
\operatorname{SAR}_{f}(\zeta)=\frac{1}{2} \frac{\sigma_{f}(\zeta)}{\rho(\zeta)} \boldsymbol{p}_{f}^{* t}\left\langle\boldsymbol{E}_{f}^{*}(\zeta), \boldsymbol{E}_{f}^{t}(\zeta)\right\rangle \boldsymbol{p}_{f}
$$

where $\langle\cdot, \cdot\rangle$ denotes the scalar product, while $\sigma_{f}$ and $\rho$ are the local material conductivity (frequency-dependent) and density, respectively. The outer product, called SAR matrix and denoted $\boldsymbol{Q}_{f}$, contains information about the complex SAR contributions from each channel irrespective of their polarization directions:

$$
\begin{aligned}
& \boldsymbol{Q}_{f}=\frac{1}{2} \frac{\sigma_{f}}{\rho}\left\langle\boldsymbol{E}_{f}^{*}, \boldsymbol{E}_{f}^{t}\right\rangle \\
& \left\langle\boldsymbol{E}_{f}^{*}, \boldsymbol{E}_{f}^{t}\right\rangle=\left(\boldsymbol{e}_{f}^{X}\right)^{*}\left(\boldsymbol{e}_{f}^{X}\right)^{t}+\left(\boldsymbol{e}_{f}^{Y}\right)^{*}\left(\boldsymbol{e}_{f}^{Y}\right)^{t}+\left(\boldsymbol{e}_{f}^{Z}\right)^{*}\left(\boldsymbol{e}_{f}^{Z}\right)^{t},
\end{aligned}
$$

where a Cartesian coordinate system has been assumed. By construction, $\boldsymbol{Q}_{f}$ is Hermitian and positive semidefinite. This property allows us to perform an inverse operation and decompose $\boldsymbol{Q}_{f}$ into an outer product, such 


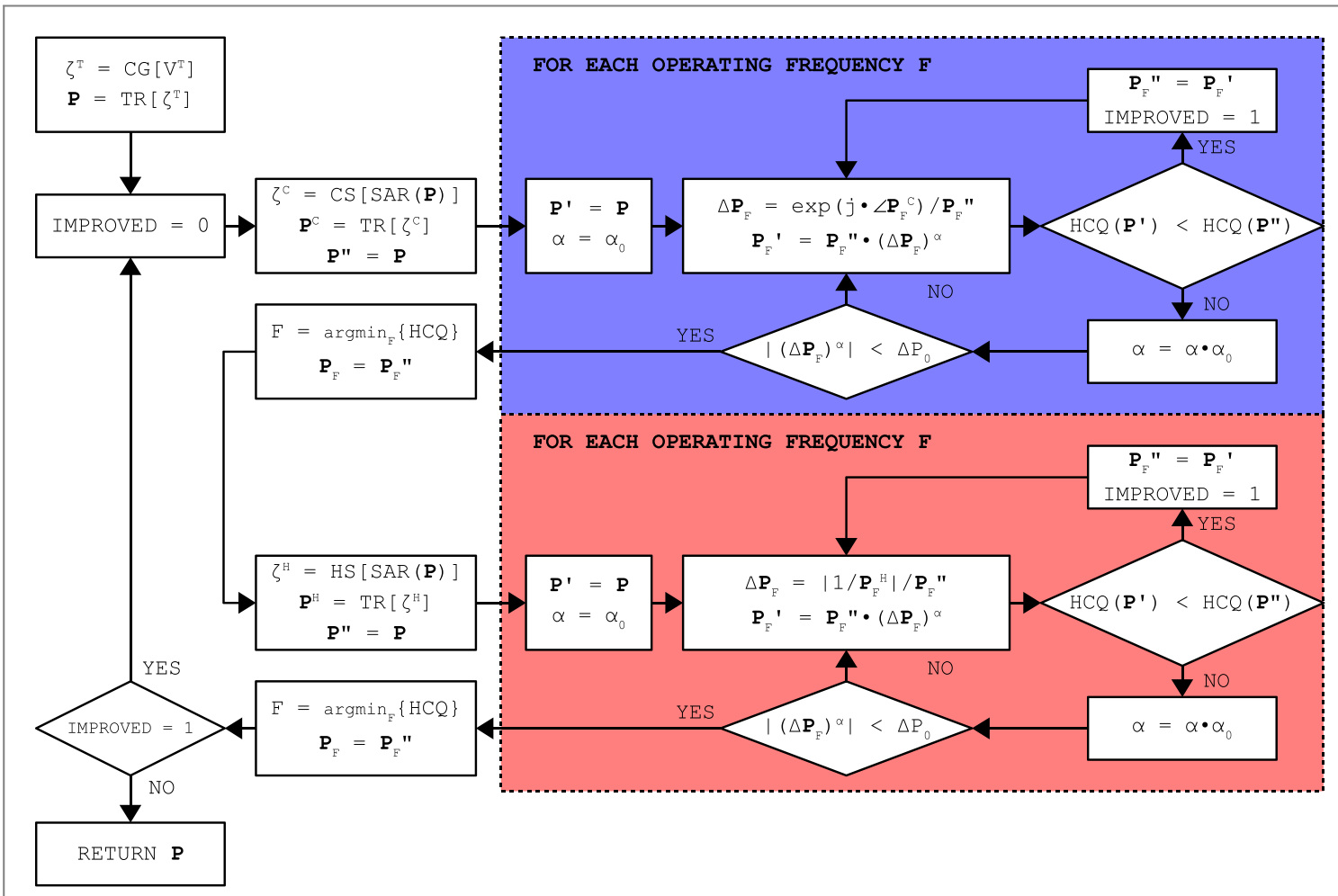

Figure 3. Schematic of the proposed i-TR focusing algorithm. CG $\left[\mathrm{V}^{\mathrm{T}}\right]$ stands for the center of gravity of the target volume. TR $[\zeta]$ indicates the TR solution obtained by placing the virtual source at $\zeta$, normalized to the amplitude of the strongest channel. The $C$ subscript refers to a cold-spot solution, and the blue section represents a cold-spot iteration. The $\mathrm{H}$ subscript refers to a hot-spot solution, and the red section represents a hot-spot iteration. The CS sub-routine determines the location of a cold-spot as the center of mass of the target sub-volume containing the lowest 1-percentile of SAR values. HS does the equivalent for the highest 1-percentile in the remaining. Within each iterative section, only the subset of steering parameters relative to the current frequency is changed, while the values relative to the other frequencies are left at the current solution P. The HCQ is always evaluated over the total SAR.

that:

$$
\boldsymbol{Q}_{f}=\hat{\boldsymbol{p}}_{f}^{*} \hat{\boldsymbol{p}}_{f}^{t}
$$

where $\hat{\boldsymbol{p}}_{f}$ is a column vector containing the complex contribution of every channel at $\zeta$. The solution $\hat{\boldsymbol{p}}_{f}$ of the decomposition can be obtained as the only non-singular eigenvector of $\boldsymbol{Q}_{f}$. The sought set of steering parameters for focusing at $\zeta$ in a generalized TR perspective is the complex conjugate of this solution:

$$
p_{c, f}=\hat{p}_{c, f}^{*} \text {. }
$$

\subsection{Iterative time-reversal}

The proposed iterative TR (i-TR) focusing technique takes into account all the points discussed above to improve an initial TR solution for HTP. The schematic of the procedure is shown in figure 3 . The initial solution $p$ is found at the center of mass of the tumor using equation (6). The resulting SAR distribution is analyzed and the most prominent cold-spot is identified as the center of mass of the contiguous sub-volume containing the lowest 1-percentile of SAR within the target volume. The TR solution at the cold-spot is calculated and the iterations begin for one frequency at a time, shifting the phase of the current parameter set towards that of the cold-spot solution $\boldsymbol{p}^{C}$.

The iterative section aims at minimizing a novel cost function specifically tailored for i-TR, which we will call the hot-to-cold spot quotient (HCQ), defined as:

$$
\mathrm{HCQ}=\frac{\overline{\mathrm{SAR}}_{\mathrm{R} 1}}{\overline{\mathrm{SAR}}_{\mathrm{T} 1}},
$$

where $\overline{\mathrm{SAR}}_{\mathrm{R} 1}$ is the average SAR in the sub-volume of remaining HSs containing the highest 1-percentile of SAR values, while $\overline{\mathrm{SAR}}_{\mathrm{T} 1}$ is the equivalent for the lowest 1-percentile in the target volume. The individual frequency iteration is carried out stepping by a geometrical factor $\alpha$, with $0<\alpha<1$ :

$$
\boldsymbol{p}_{f}^{\prime}=\boldsymbol{p}_{f}^{\prime \prime} \cdot \Delta \boldsymbol{p}_{f}^{\alpha},
$$




$$
\Delta \boldsymbol{p}_{f}=\left\{\begin{array}{l}
\left(e^{j<p_{f}^{C}}\right) / \boldsymbol{p}_{f}^{\prime \prime}, \text { for cold-spot iteration } \\
\left(\left|1 / \boldsymbol{p}_{f}^{H}\right|\right) / \boldsymbol{p}_{f}^{\prime \prime}, \text { for hot-spot iteration }
\end{array},\right.
$$

where $j$ is the imaginary unit, and all operations are intended to be element-wise. The buffer variable $\boldsymbol{p}_{f}^{\prime \prime}$, initially set as $\boldsymbol{p}_{f}$, is replaced by the search direction $\boldsymbol{p}_{f}^{\prime}$ only if the stepping was successful, i.e. if the resulting $\operatorname{HCQ}\left(\boldsymbol{p}^{\prime}\right)$ is better than the current $\operatorname{HCQ}\left(\boldsymbol{p}^{\prime \prime}\right)$. Note that, while only the subset of steering parameters relative to one frequency is iterated at a time, the assessment of HSs, cold-spots and HCQ at each iteration is always carried out over the total SAR distribution:

$$
\mathrm{SAR}=\mathrm{SAR}_{1}+\mathrm{SAR}_{2}+\ldots+\mathrm{SAR}_{n_{f}} .
$$

If the stepping was not successfull, the step factor $\alpha$ is geometrically reduced and another attempt is made. If the norm of the step $\left|\Delta \boldsymbol{p}^{\alpha}\right|$ falls below a threshold value $\Delta p_{0}$ without any improvement on the HCQ, the next frequency is considered and iterated. Once all frequencies have been iterated, only the subset of parameters $\boldsymbol{p}_{f}$ relative to the frequency that yielded the best improvement in HCQ is updated with $\boldsymbol{p}_{f}^{\prime \prime}$.

A similar procedure is then carried out for the HS. The updated SAR distribution is analyzed and the most prominent HS is identified as the highest 1-percentile of SAR within the HSs. However this time the amplitude of the parameters is shifted away from the HS solution $\boldsymbol{p}^{H}$. If at least one spot iteration was successful in improving the HCQ, a new cold-spot is considered and iterated, and so on. Otherwise, the algorithm terminates.

The parameter $\Delta p_{0}$ is directly related to the desired precision on the steering parameters. We select a precision of $1 \%\left(\Delta p_{0}=0.01\right)$ since RF systems usually do not provide steering accuracy higher than $\pm 5 \%$ (Wust et al 1998, Paulides et al 2007). The parameter $\alpha_{0}$, on the other hand, decides the length of the first iterative step and its subsequent geometrical progression. As shown later in section 4.3, the value of $\alpha_{0}$ affects mainly the algorithm's convergence speed and number of iterations, and has almost no effect on the overall accuracy. Its optimal value $\alpha_{0}=0.1$ is determined by means of a sensitivity analysis, and this value is kept throughout all executions reported here.

\section{Method}

We compare the proposed i-TR technique with EV, PS, and classic TR. The evaluation is carried out in two steps: we first assess the quality of the HTPs via SAR-based indicators, then validate them in terms of resulting thermal distributions. The test cases include 3 inhomogeneous artificial targets of increasing size in the neck region and 2 tumor-filled realistic targets, one in the neck and one in the brain. The thermal validation is carried out only for the realistic targets. The HTP methods are benchmarked in terms of the following quality indicators:

- HTQ: HS to target quotient, as defined in Canters et al (2010):

$$
\mathrm{HTQ}=\frac{\overline{\mathrm{SAR}}_{\mathrm{R} 1}}{\overline{\mathrm{SAR}}_{\mathrm{T}}}
$$

i.e. the ratio between the average SAR in the sub-volume containing the highest 1-percentile SAR among the remaining tissues $\left(\overline{\mathrm{SAR}}_{\mathrm{R} 1}\right)$ and the average SAR in the target volume $\left(\overline{\mathrm{SAR}}_{\mathrm{T}}\right)$. Values of HTQ around or below 1 are typically considered for clinical treatment.

- $\mathrm{TC}_{\mathrm{x}}$ : iso-SAR target coverage, as defined in Togni et al (2013):

$$
\mathrm{TC}_{x}=\frac{V_{\mathrm{T}}(x)}{V_{\mathrm{T}}}, \quad V_{\mathrm{T}}(x) \mid \operatorname{SAR}\left[V_{\mathrm{T}}(x)\right] \geqslant x \cdot \operatorname{SAR}[V]
$$

i.e. the fraction of the target volume $V_{\mathrm{T}}$ where SAR values are above a given fraction $x$ of the SAR peak value in the whole patient volume $V$. Both the $\mathrm{TC}_{x}$ value and the fraction $x$ are usually expressed as percentage, and the $\mathrm{TC}_{x}$ index is evaluated in this work for $x=25 \%$ or $x=50 \%$ depending on the model at hand. Values of $\mathrm{TC}_{25}$ greater than $75 \%$ are typically considered for clinical treatment.

Both the HTQ and TC indicators are evaluated over the $1 \mathrm{~g}$-averaged SAR distribution as detailed in section 3.3. The TR, i-TR, EV and PS focusing algorithms are all implemented in MATLAB ${ }^{\circledR}$ (The MathWorks Inc. 2019). Field processing steps are parallelized and executed in single precision on a high performance GPU (nVidia Quadro RTX 6000) where possible. We further evaluate the speed and computational cost of each method using the following metrics:

- Running time, $t_{r}$ : time needed for the HTP optimization algorithm to complete, excluding SAR matrix preparations. 


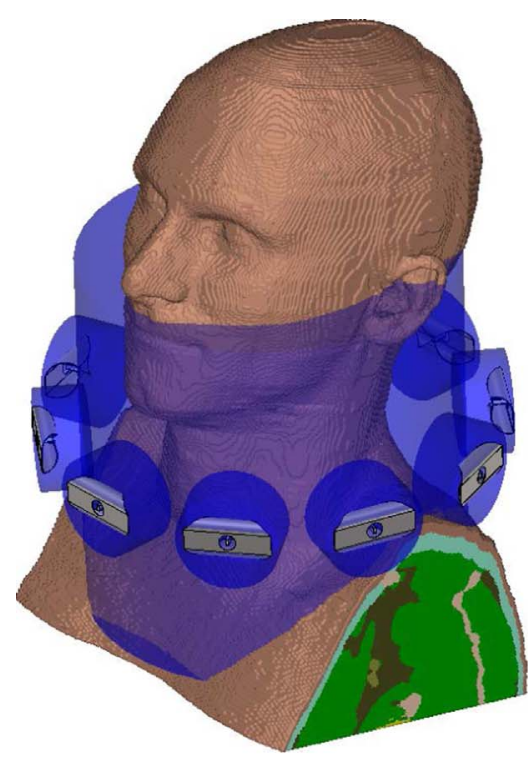

(a) Collar applicator, neck.

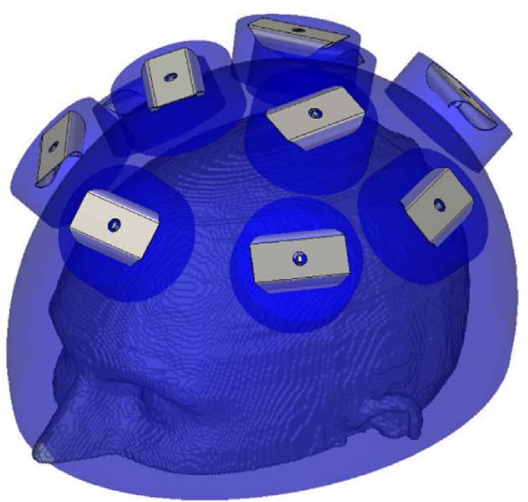

(b) Helmet applicator, brain.

Figure 4. The simulation setups for the collar (a) and the helmet (b) applicators, consisting of patient voxel model, water bolus, and 10 SGBT antennas each.

- Number of evaluations, $n_{e}$ : how many times the cost function is evaluated by the algorithm (zero for EV and classic TR).

For the thermal validation, whose setup is detailed in section 3.4, the following HTP quality indicators are used to evaluate the resulting temperature distributions:

- Cumulative histograms: of the temperature distribution within the target.

- $\mathrm{T}_{50}$ : median temperature in the target volume, as defined in Fatehi et al (2007).

\subsection{Applicator array topologies}

The HTP algorithms are tested on two array topologies: cylindrical and semi-spherical. The cylindrical applicator (figure 4(a)) is meant for treatment of tumors in the neck region. It consists of 10 radiators arranged on one ring of $20 \mathrm{~cm}$ diameter. The radiators are self-grounded bow-tie antennas (SGBT), which work across the range 400-800 MHz and have been adapted specifically for ultra-wideband MW-HT (Takook et al 2017). A cylindrical water bolus, with average thickness $4 \mathrm{~cm}$, is included between the antennas and the patient to improve matching. The SGBT antennas are further immersed each into a protruding cylindrical extension of the water bolus, to enhance their directivity and reduce cross-coupling between array elements.

The semi-spherical applicator (figure 4(b)) represents a simplified version of a helmet applicator for brain tumors. It consists of 10 SGBT antennas radially arranged from the top of the scalp and following the skull's curvature. The applicator covers only a small part of the head and, as such, can only be meaningfully used for targeting volumes close to the the skull. This applicator is not optimized for an effective treatment of deep-seated brain tumors, but it is intended for the investigation of the i-TR method's robustness to mixed polarization axes. The average water bolus thickness for this applicator is $1.5 \mathrm{~cm}$.

\subsection{Human model and target volumes}

The Duke human voxel model from the IT'IS Foundation (Gosselin et al 2014) is used as virtual patient for both regional treatment plannings. The original resolution of $1 \times 1 \times 1 \mathrm{~mm}$ is down-sampled to $2 \times 2 \times 2 \mathrm{~mm}$ to spare computational resources. The constant (density) and dispersive (permittivity, conductivity) tissue properties are obtained from the IT'IS Foundation database (Hasgall et al 2012). A $230 \times 260 \times 390 \mathrm{~mm}$ subset of the model is selected to represent the neck region, figure 4 (a). The resulting voxel matrix includes 47 segmented tissues. For the brain applicator, a smaller subset is selected, $230 \times 260 \times 138 \mathrm{~mm}$, figure 4 (b). The resulting voxel matrix includes 31 tissues. 


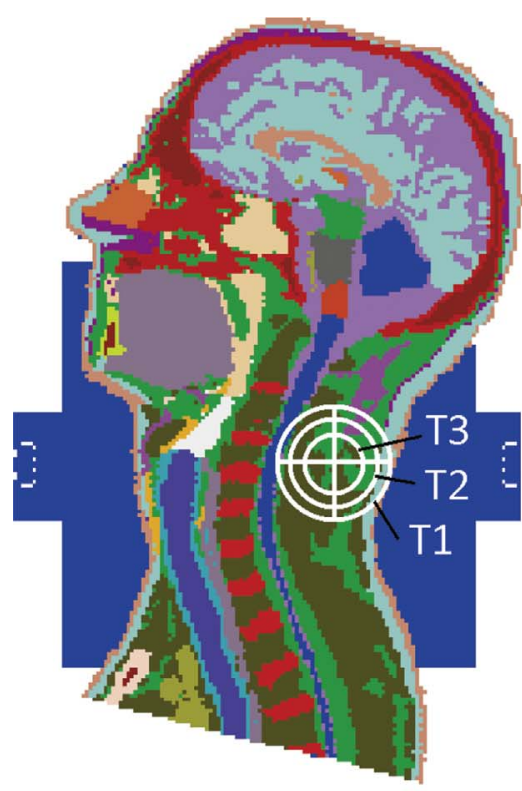

(a) Dorsal, $T_{1,2,3}$, sagittal.

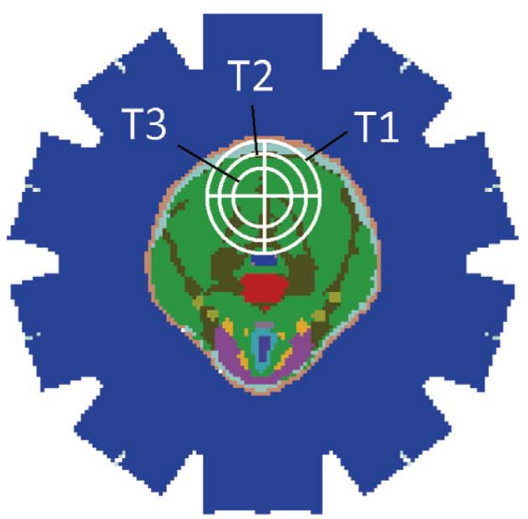

(b) Dorsal, $T_{1,2,3}$, transverse.

Figure 5. Artificial heterogeneous target volumes. The targets are spherical and concentric. They are delineated in white. The volume center is indicated with a cross. Sections are taken at the volume center.

\begin{tabular}{|c|c|c|c|c|c|}
\hline Label & Region & Volume & Location & Topology & Composition \\
\hline$T_{1}$ & Neck & $113 \mathrm{ml}$ & Dorsal & Spherical & $73 \% \mathrm{M}, 18 \% \mathrm{~F}, 09 \% \mathrm{O}$ \\
\hline$T_{2}$ & Neck & $48 \mathrm{ml}$ & Dorsal & Spherical & $77 \% \mathrm{M}, 21 \% \mathrm{~F}, 02 \% \mathrm{O}$ \\
\hline$T_{3}$ & Neck & $14 \mathrm{ml}$ & Dorsal & Spherical & $78 \% \mathrm{M}, 22 \% \mathrm{~F}, 00 \% \mathrm{O}$ \\
\hline$T_{4}$ & Neck & $85 \mathrm{ml}$ & Larynx & Concave & $25 \% \mathrm{X}, 20 \% \mathrm{M}, 18 \% \mathrm{~L}, 13 \% \mathrm{~T}$ \\
\hline$T_{5}$ & Brain & $30 \mathrm{ml}$ & Meninges & Convex & $46 \%$ C, $28 \%$ G, $22 \% \mathrm{~W}, 04 \% \mathrm{O}$ \\
\hline
\end{tabular}

For the preliminary SAR investigation, three spherical target volumes are defined in MATLAB ${ }^{\circledR}$ after the E-field simulations. As the original dielectric properties within the target volumes are kept, these targets are heterogeneous. In addition to these, two realistic test cases are considered: one in the larynx for the neck applicator and one in the meninges for the brain applicator. These targets are defined before the E-field simulations, and their volumes are filled with a homogeneous material whose properties are determined by taking the weighed average of the materials originally inside. This results in a conservative approach where the tumor does not exhibit increased conductivity. The volume and composition of the 5 targets are described in table 1 and shown in figures 5 and 6. Resulting tumor properties for the realistic cases are reported in table 2.

In all cases, the target volume is a contiguous shape, as multi-target treatment planning is beyond the scope of the present work. The artificial targets are included to evaluate the i-TR scheme's ability to achieve target coverage and HS suppression for different tumor sizes. The larynx case is included to assess the HTP's ability to target concave shapes with both superficial and deep areas. Note that the trachea lumen creates a cavity in the target volume. The meningioma is included to investigate the case where multiple polarization directions are interfering in the target volume.

\subsection{E-field simulations and SAR computation}

The E-field distributions of each antenna within the applicator are obtained numerically using the commercial software CST Microwave Studio (Dassault Systèmes SE, Vélizy-Villacoublay, France 2019). The software implements an FDTD scheme on a hexahedral mesh with possibility for locally denser sampling grids. The complex geometrical curves of the SGBT antennas are sampled at $0.5 / \sqrt{3} \mathrm{~mm}$ to correctly represent at any angle the $0.5 \mathrm{~mm}$ thick copper plate with which they are realized. The mesh resolution within the patient varies between this value and a maximum of $2 \mathrm{~mm}$. Together with the water bolus, this results in $\approx 125 / 220$ million 


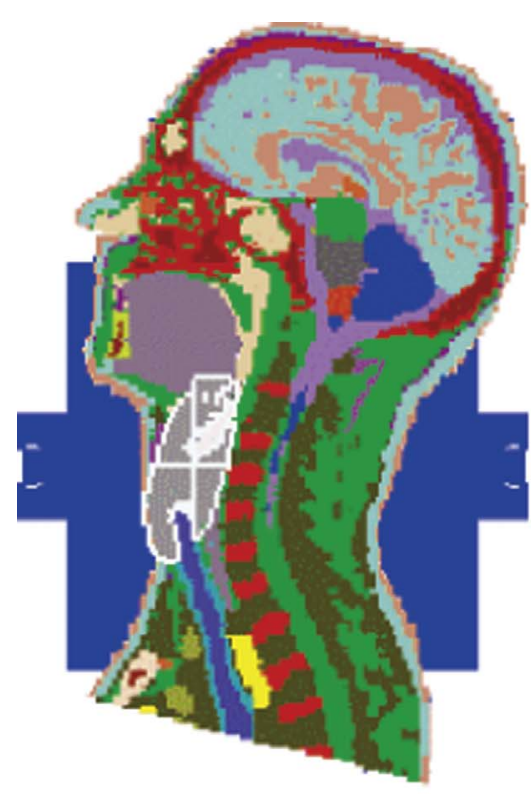

(a) Larynx, $T_{4}$, sagittal.

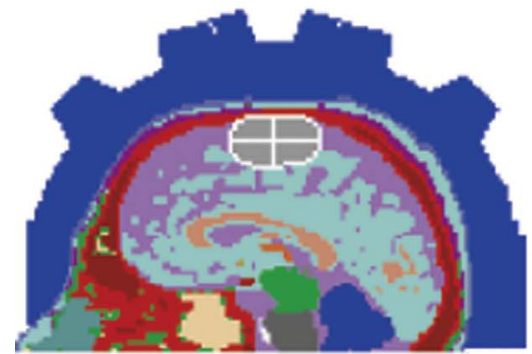

(c) Meninges, $T_{5}$, sagittal.

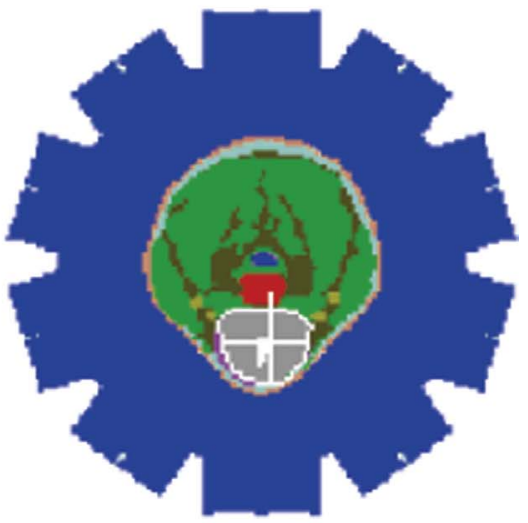

(b) Larynx, $T_{4}$, transverse.

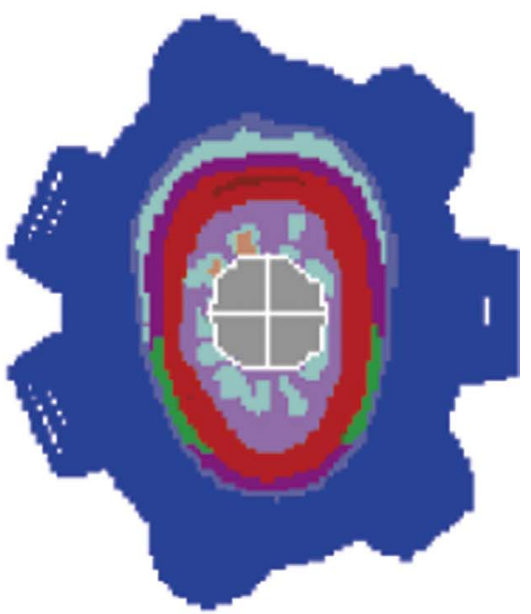

(d) Meninges, $T_{5}$, transverse.

Figure 6. Realistic homogeneous target volumes. The targets are delineated in white. The volume center is indicated with a cross. Sections are taken at the volume center.

Table 2. Properties of the homogeneous materials filling the target volumes in the realistic cases, obtained as the weighed average of the original materials within the volume. Some properties are adjusted for hyperthermic conditions.

\begin{tabular}{lccccccc}
\hline Tumor & $\epsilon[\sim]$ & $\sigma\left(\mathrm{S} \mathrm{m}^{-1}\right)$ & $\rho\left(\mathrm{kg} \mathrm{m}^{-3}\right)$ & $k\left(\mathrm{~W} \mathrm{Km}^{-1}\right)$ & $c\left(\mathrm{~K} \mathrm{~K}^{-1} \mathrm{Kg}^{-1}\right)$ & $\omega_{b}\left(\mathrm{~kW} \mathrm{Km}^{-3}\right)$ & $Q_{m}\left(\mathrm{~kW} \mathrm{~m}^{-3}\right)$ \\
\hline Larynx & 47.8 & 0.674 & 1088 & 0.423 & 3.285 & 22.033 & 5.456 \\
Meninges & 57.3 & 1.372 & 1063 & 0.535 & 3.754 & 17.048 & 5.478 \\
\hline
\end{tabular}

cells for the collar/helmet setups, respectively. The almost doubled amount of mesh cells for the helmet setup is due to the antenna arrangement occupying more effectively all 3 dimensions in space. Absorbing boundary conditions (PML) are defined for all sides of the computational domain.

Upon import for post-processing in MATLAB ${ }^{\circledR}$, the E-field distributions are re-sampled to a constant grid resolution of $2 \mathrm{~mm}$. Out of these distributions, the local SAR matrices $Q_{f}(x, y, z)$ are constructed according to equation (4). This results in 1.1 million matrices for the neck model and 0.3 million for the brain one (considering the patient only). Each element $q_{i, j}(x, y, z)$ is further spatially averaged according to a $1 g$ SAR massaveraging scheme. The averaging scheme is similar to the CST Legacy one as described in (Qi et al 2008): we treat surface voxels by expanding a spherical kernel until the mass of patient tissues within reaches $1 \mathrm{~g}$. 


\subsection{Thermal simulations}

To validate the HTP plans, steady-state thermal simulations are performed with CST Microwave Studio ${ }^{\circledR}$. We consider only the realistic scenarios and only the frequency combination that yields the lowest HTQ after i-TR optimization. This combination would be the one selected for treatment after quickly surveying all possible combinations with i-TR. The thermal properties of the HSs are also taken from the IT'IS database. However, to mimic the hyperthermic stress condition of the body, the muscle's blood flow perfusion is increased by a factor 4 (Lang et al 1999, De Greef et al 2010). Moreover, the thermal conductivity of the cerebrospinal fluid is increased by a factor 10 to emulate the convective transport of heat (Schooneveldt et al 2019). The tumor thermal properties are calculated as a weighed average of the tissues originally composing its volume, in the same way as for the electromagnetic properties. The tumor blood perfusion rate is further diminished by a factor 0.7 to account for the chaotic vascular structure that characterizes neoplasms (Song 1984, Lang et al 1999). The tumor properties are summarized in table 2 .

\subsection{Choice of operating frequencies}

The set of possible operating frequencies is constructed by stepping $100 \mathrm{MHz}$ within the antenna working range 400-800 MHz, yielding the following set:

$$
F=[400,500,600,700,800]^{t} \mathrm{MHz} .
$$

Out of this set, all the single and double frequency combinations are evaluated, resulting in 15 operating frequency settings. This is to highlight potential frequency-dependent phenomena within the patient and to evaluate the i-TR scheme's robustness to different combinations of operating frequencies, while at the same time keeping the amount of test cases at a minimum.

\subsection{Implementation of EV and PS}

The EV implementation is similar to the one used in Guérin et al (2018). It determines via a direct solution the set of steering parameters that maximizes the SAR amplification factor (SAF), defined as the following quadratic ratio:

$$
\mathrm{SAF}=\frac{\overline{\mathrm{SAR}}_{\mathrm{T}}}{\overline{\mathrm{SAR}}_{\mathrm{R}}},
$$

where $\overline{\mathrm{SAR}}_{\mathrm{T}}$ is the average SAR in the target, and $\overline{\mathrm{SAR}}_{\mathrm{R}}$ is the average SAR in the rest. An important consequence of how equation (14) is constructed is that only one frequency at a time from any working set can maximize such ratio (Zanoli and Trefná 2020). The amplitude of the other frequencies must be set to zero, and the only active frequency is the one whose interference pattern best fits the target shape and location. This approach differs substantially from the continuous FIR solution described in Converse et al (2006), which, to the best of the authors' knowledge, is the only published study on wide-band EV beam-forming. Their approach is not applicable here since the spectrum is discrete. Naturally, maximizing the SAF is not the same as minimizing nonlinear indicators such as HTQ, which usually correlate better with the resulting thermal dose.

The PS implementation used for this study is the one readily available in MATLAB ${ }^{\circledast}$ (particleswarm). The cost function is the HTQ as described at the beginning of this section. In order for PS to properly converge regardless of the problem's complexity (Piotrowski et al 2020), some of its settings are made proportional to the number of variables, $n_{v}=2 \cdot n_{c} \cdot n_{f}$, i.e. phase and amplitude for each channel at each frequency. In particular, the number of particles is set to $10 \cdot n_{v}$, and the optimization is halted when $n_{v}$ consecutive iterations have produced a relative change in the objective function smaller than 0.1 . Other settings have been kept to their default values. When the partic leswarm algorithm has completed, the optimization is handed over to a local optimizer (fmincon) and the solution is further refined until the relative parameter change falls below 0.01 , thus reaching the desired precision of $1 \%$.

\section{Results}

Figure 7 shows a typical i-TR processing and its results for a representative artificial target case. Starting from the classic TR solution, the HCQ is progressively minimized until no further improvement is achieved by either cold- or $\mathrm{HS}$ iterations. During this process, the $\mathrm{HTQ}$ and $\mathrm{TC}_{25}$ vary according to the current solution. In general, $\mathrm{HTQ}$ floats around the initial value, while $\mathrm{TC}_{25}$ is strongly enhanced within a few iterations. The HCQ value is improved significantly throughout the entire process. This reflects in a more homogeneous SAR distribution within the target and in a reduction of the most relevant HS, which, in this case, is located in the skin layer adjacent to the target. The initial steering parameter amplitudes, figure 7, exhibit the typical TR pattern where each antenna radiates power proportionally to its vicinity to the target. The final amplitude settings clearly 


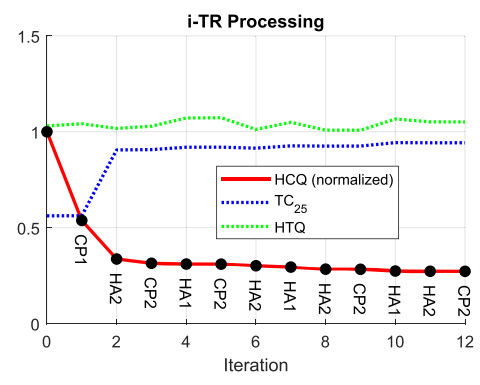

(a)
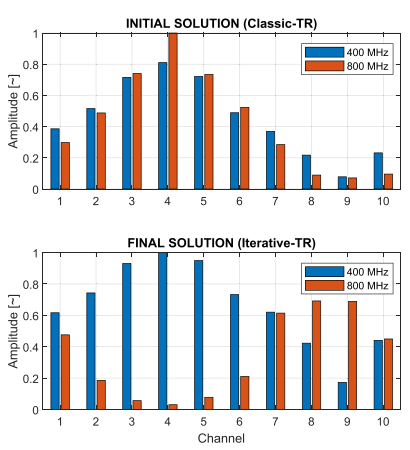

(b)

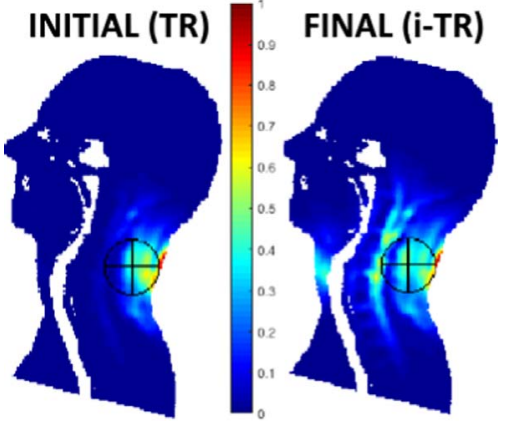

(c)

Figure 7. Example of i-TR processing of a double-frequency problem, $400+800 \mathrm{MHz}$. $T_{1}$ artificial heterogeneous target. (a) values of HCQ (normalized to the initial value), $\mathrm{HTQ}$ and $\mathrm{TC}_{25}$ at each iteration. Black dot labels report which spot has caused the improvement: CPn cold-spot/phase at frequency n, HAn hot-spot/amplitude. (b) initial and final steering amplitudes for all channels. (c) Corresponding SAR distributions (normalized to the highest value). Sagittal section at target center.

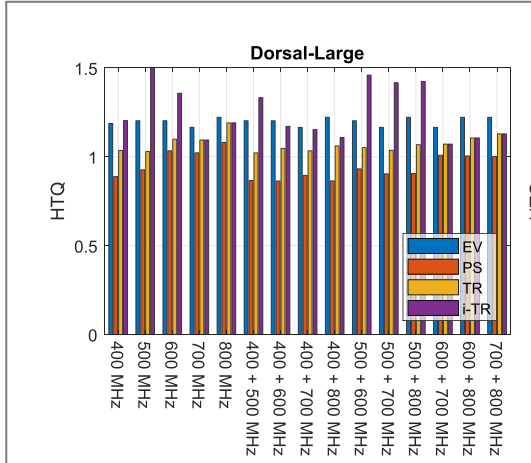

(a) Target $T_{1}$

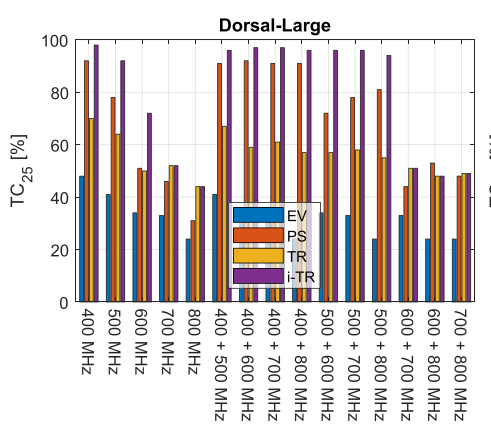

(d) Target $T_{1}$.

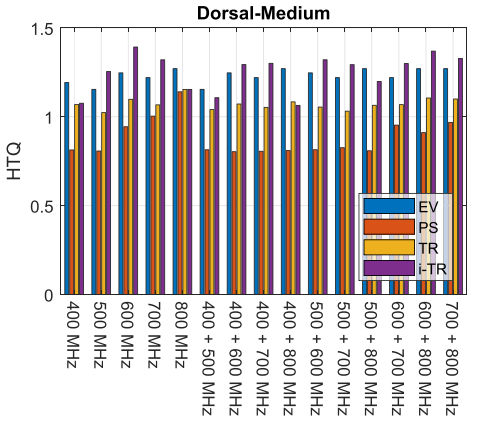

(b) Target $T_{2}$

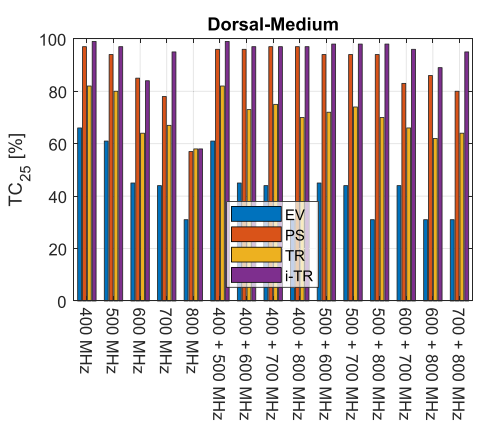

(e) Target $T_{2}$.

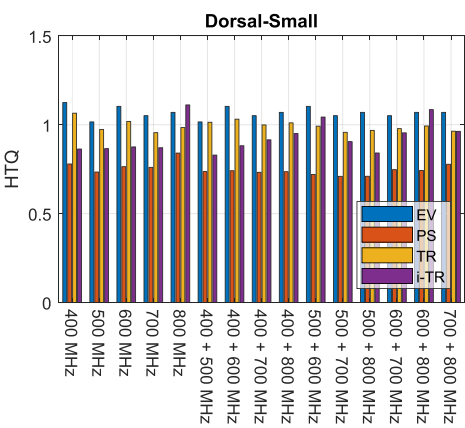

(c) Target $T_{3}$

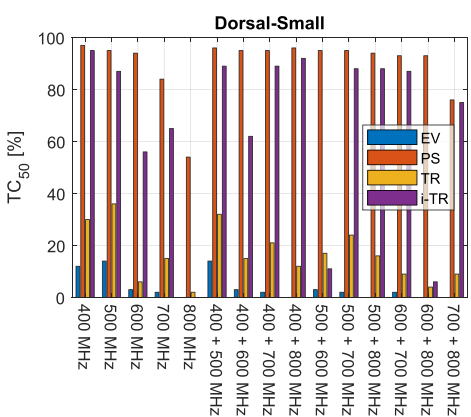

(f) Target $T_{3}$

Figure 8. SAR-based HTP quality indicators in the artificial test cases, for all operating frequency combinations. (a)-(c) report the HTQ while (d)-(f) report target coverage. $\mathrm{TC}_{50}$ is shown in (f) due to $\mathrm{TC}_{25}$ being $100 \%$ in $T_{3}$.

demonstrate how i-TR can exploit different frequencies to cover both superficial and deeper parts of the target volume, as confirmed also by the SAR distribution in figure 7 (c).

\subsection{SAR evaluation}

SAR-based HTP quality indicators for the artifical test cases are reported in figure 8. The lowest HTQ for any given problem and frequency combination is achieved by PS, being configured to minimize this indicator. However, this does not always translate into better coverage. The i-TR returns sensibly higher $\mathrm{TC}_{25}$ values in the large and medium cases, at the expense of an increase in HTQ with respect to the initial TR solution. While some solutions become impractical due to this increase, there is always at least one optimal frequency combination 


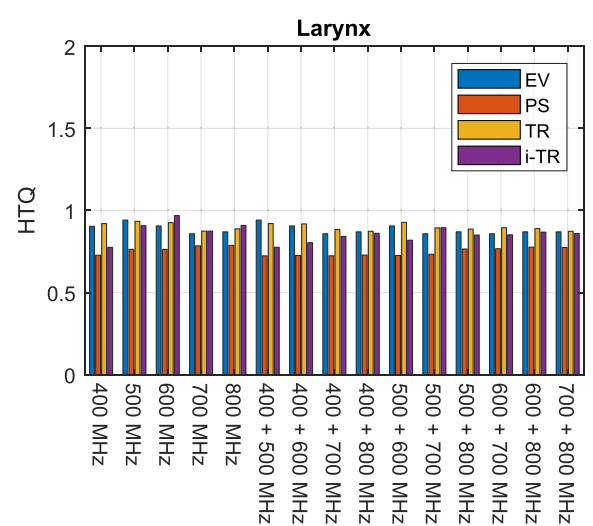

(a) Target $T_{4}$

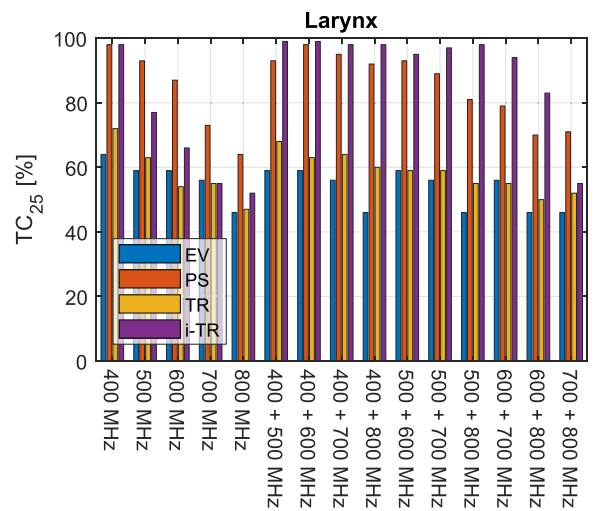

(c) Target $T_{4}$

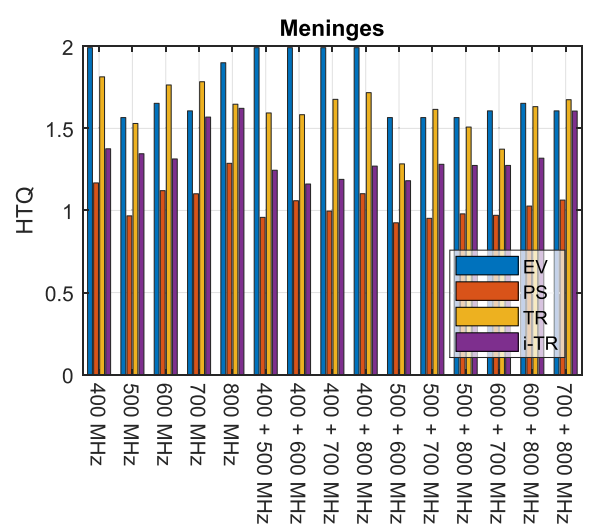

(b) Target $T_{5}$

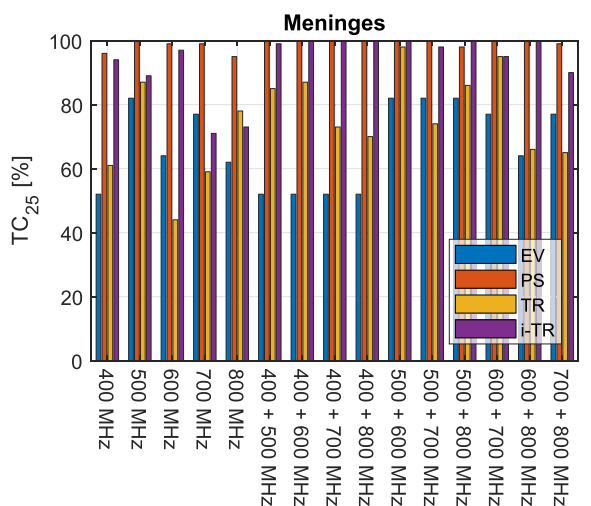

(d) Target $T_{5}$

Figure 9. SAR-based HTP quality indicators in the realistic test cases, for all operating frequency combinations. (a) and (b) report the HTQ while (c) and (d) report target coverage.

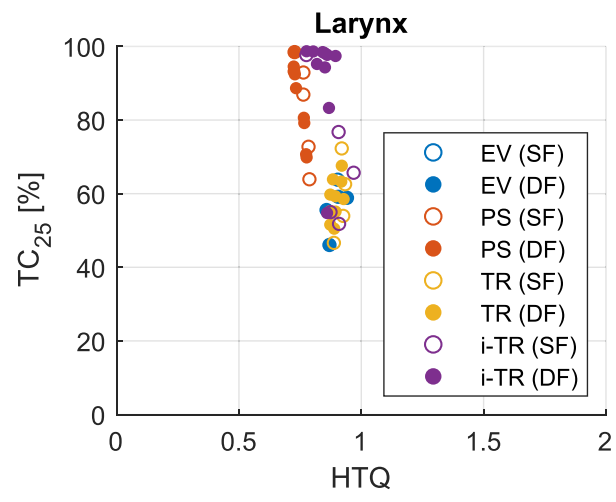

(a) Target $T_{4}$.

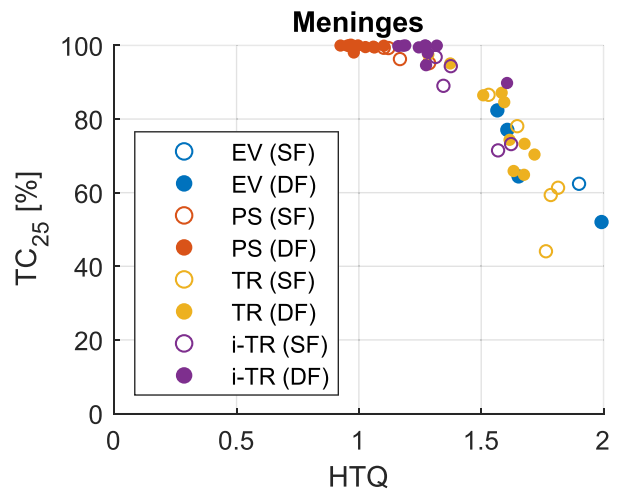

(b) Target $T_{5}$.

Figure 10. Combined HTQ-TC 25 plots for the HTP solutions in the realistic test cases. Empty circles denote single-frequency (SF) solutions. Filled circles denote dual-frequency (DF) solutions.

where $\mathrm{i}$-TR achieves $\mathrm{HTQ} \approx 1$ and $\mathrm{TC}_{25} \approx 100 \%$. In the small target case, $\mathrm{i}$-TR also lowers the HTQ provided by classic TR while achieving remarkably higher $\mathrm{TC}_{50}$ values. For this target, which is less heterogeneous than $T_{1}$ and $T_{2}$ (see table 1 ), the solutions provided by PS and i-TR are comparable in quality. EV exhibits consistently high HTQ and poor coverage in all cases.

SAR-based HTP indicators for the realistic targets are reported in figure 9. Once more, PS returns the lowest HTQ for each case. All algorithms yield comparable HTQ values below 1 in the larynx, while only PS manages to reach values below 1 in the meninges. EV and classic TR perform similarly in the larynx case, achieving 


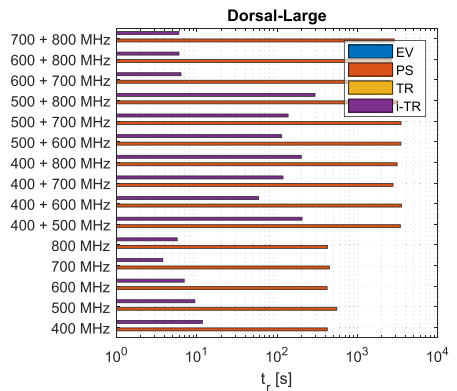

(a) Target $T_{1}$

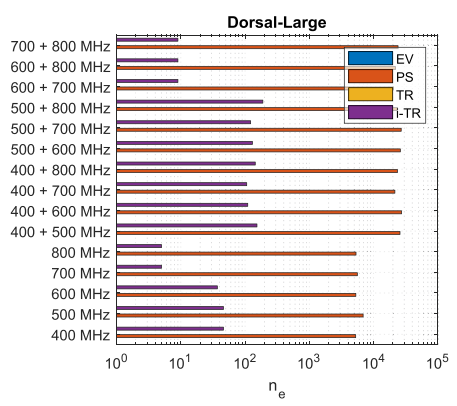

(d) Target $T_{1}$

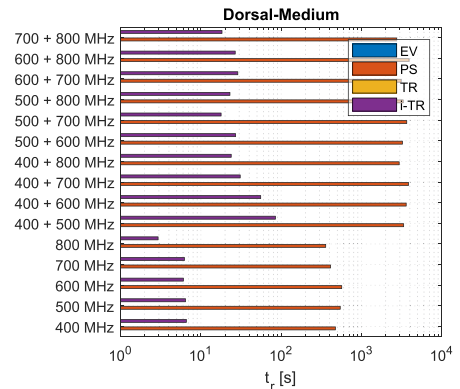

(b) Target $T_{2}$

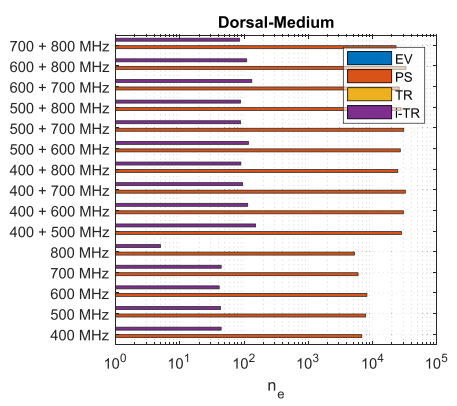

(e) Target $T_{2}$.

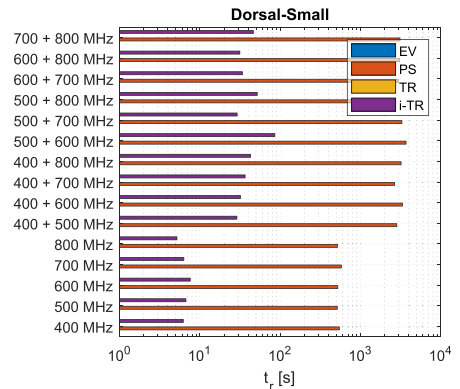

(c) Target $T_{3}$

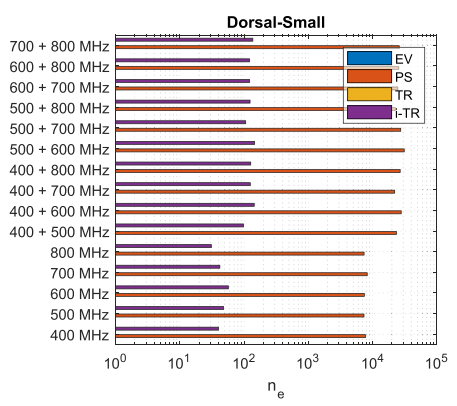

(f) Target $T_{3}$.

Figure 11. Algorithm speed performance metrics in the artificial test cases, for all operating frequency combinations, on a X-logarithmic scale. (a)-(c) report the running time while (d)-(f) report the number of cost function evaluations. $t_{e} \approx 0.5 \mathrm{~s}$ and $n_{e}=0$ for both EV and classic TR since they are direct solution methods.

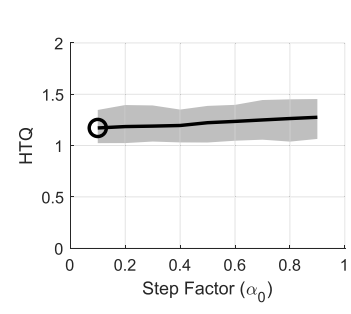

(a)

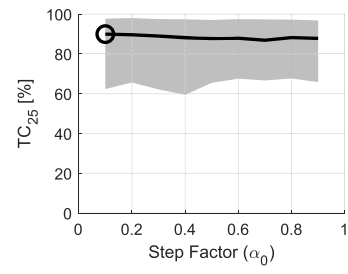

(b)

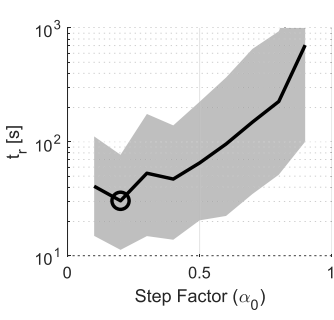

(c)

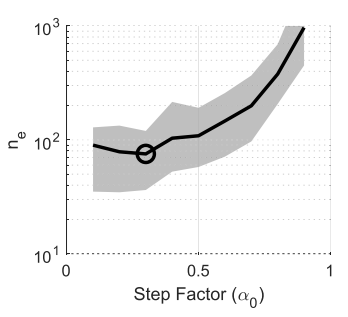

(d)

Figure 12. Analysis of the i-TR algorithm's sensitivity to the stepping parameter $\alpha_{0}$. The plots report mean (black line) and standard deviation (gray shade) of all the artificial test cases for all frequency combinations $(n=45)$. The black circle indicates the optimal value across a parametric sweep. (a) and (b) report the SAR-based HTP quality indicators while (c) and (d) report the performance metrics (Y-log plot).

acceptable HTQ, but suboptimal coverage. EV fails to provide viable solutions for the meninges, with HTQ always above 1.5, and $\mathrm{TC}_{25}$ above threshold only at $500 \mathrm{MHz}$ and $700 \mathrm{MHz}$. In contrast, i-TR yields HTQ values much closer to the global PS optimum for this case. More importantly, it does so with simultaneous extensive target coverage. In the larynx model, $\mathrm{TC}_{25}$ is often higher for $\mathrm{i}$-TR than for PS when more than one frequency is considered. The SAR results for these two realistic cases are further summarized into two combined HCQ-TC 25 plots in figure 10. In both plots, the dual-frequency solutions provided by i-TR are seen to be located near the $\mathrm{TC}_{25}$ optimum (100\%) and close to PS's global HTQ optimum.

\subsection{Performance analysis}

Speed performance metrics for the artificial test cases are reported in figure 11 . Since EV and TR are direct solution methods, they take less than a second to complete. About 6000 cost function evaluations are needed for PS to return a single-frequency solution, and up to 30000 in the case of two frequencies. This translates into long execution times, with a typical run taking about 10 minutes for one frequency and 50 minutes for two frequencies. Conversely, i-TR manages to return a single-frequency solution within at most 50 evaluations, and a double-frequency solution in 100 evaluations. This reflects into at most 10 s running time in the single- 


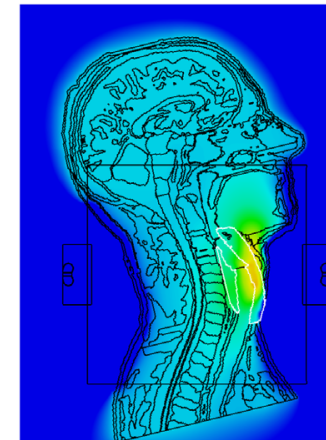

(a) EV.

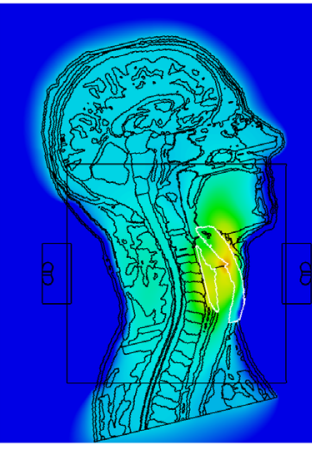

(b) PS.

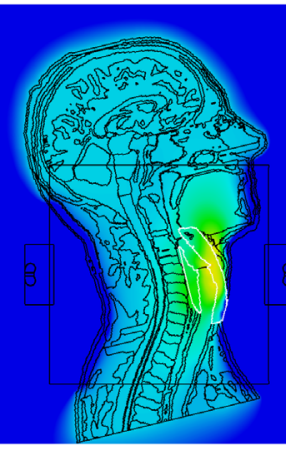

(c) TR.

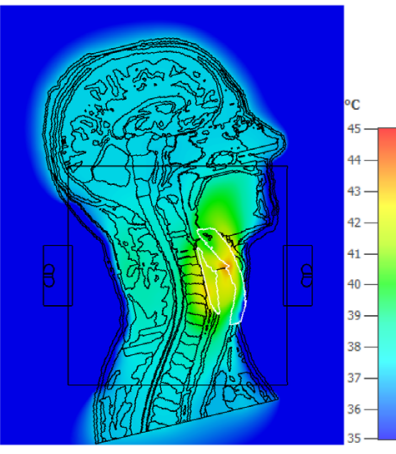

(d) i-TR.

Figure 13. Temperature distributions in the larynx realistic case for all HTP algorithms at $400+500 \mathrm{MHz}$. Maximum temperature in healthy tissues is $43^{\circ} \mathrm{C}$ in all cases. The target volume is outlined in white. Sagittal section at tumor center.

frequency case, and on average 50 seconds for a double-frequency problem. Performance metrics for the realistic cases are not reported since they indicate similar trends.

\subsection{Sensitivity analysis}

The results of the sensitivity analysis with respect to the initial step factor $\alpha_{0}$ are reported in figure 12 . Both HTP quality indicators exhibit negligible variations across the sweep, meaning that the solution provided by $\mathrm{i}$-TR is stable in this respect. A closer look suggests that the optimum is located at the lowest end of the $\alpha_{0}$ range. This can be intuitively explained by the fact that smaller stepping allows the algorithm to get closer to the HCQ optimum. High $\alpha_{0}$ values exponentially increase the convergence time and computational burden of the algorithm, indicating that the initial classic TR solution is already close to the optimum and only small steps are needed to improve its HCQ value. On the other hand, both $t_{r}$ and $n_{e}$ tend to increase again towards $\alpha_{0}=0$, as a consequence of the step being too small to quickly reach the optimum HCQ. In conclusion, $\alpha_{0}=0.1$ seems to be an appropriate value for this parameter.

\subsection{Thermal validation}

Figures 13 and 14 show the thermal distributions for the realistic cases. It can be seen that both direct solvers, EV and TR, fail to reach therapeutic temperatures in the deeper parts of the tumors. In the larynx case, the trachea acts as a heat barrier between opposing sides of the target volume, whose morphology is toroidal. PS and i-TR manage to overcome this barrier by extending the SAR coverage to the inner side, at the expense of higher average temperatures in the healthy tissues. This higher power deposition, however, does not result in additional HSs. i-TR performs better in this sense than PS by gaining half a degree in median temperature, see table 3 . For the meningioma, both EV and TR end up maximizing the peak SAR in the target which results in a focal spot close to the skull. This spot is however mitigated by the cooling effect of the bolus, so that neither high peak 


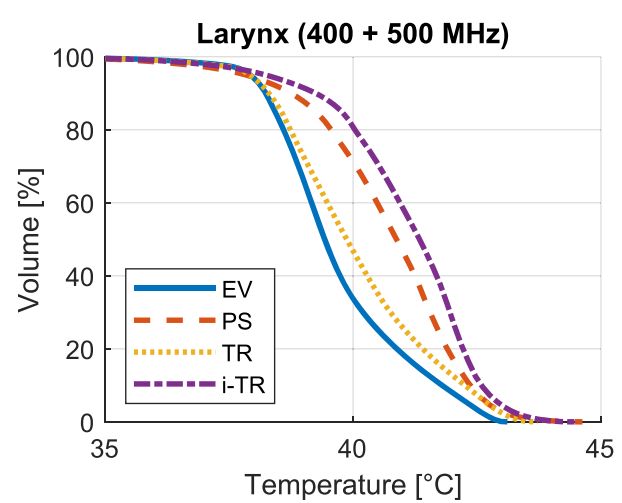

(a)

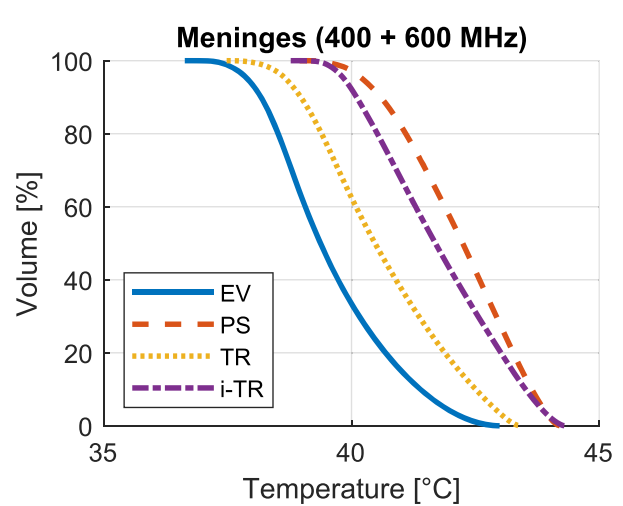

(b)

Figure 15. Cumulative histograms of the temperature distribution within the targets.

Table 3. Median temperatures $\left(T_{50}\right)$ reached inside the target volume in each of the thermal test cases for all HTP algorithms. Values in ${ }^{\circ} \mathrm{C}$.

\begin{tabular}{lcccr}
\hline Tumor & EV & PS & TR & i-TR \\
\hline Larynx & 39.43 & 40.87 & 39.87 & 41.34 \\
Meninges & 39.37 & 42.24 & 40.47 & 41.69 \\
\hline
\end{tabular}

temperatures nor sufficient coverage are achieved within the target. Both PS and i-TR shift this main SAR peak deeper in the brain, reaching high median temperatures. i-TR closely follows PS by only half a degree lower $T_{50}$. These results are further illustrated in the cumulative histograms, figure 15.

\section{Discussion}

Hyperthermia requires an optimal SAR distribution in the patient in terms of raw target power deposition, target coverage, and HS suppression in healthy tissues. Target SAR coverage has in fact been shown to be predictive for the therapeutic outcome of the treatment (Myerson et al 1990, Lee et al 1998). To improve on these aspects, the HTQ and the TCx indicators have been proposed and shown to be effective when applied retrospectively to clinical data sets (Paulides et al 2016, Bellizzi et al 2019). However, HTQ and $\mathrm{TC}_{x}$ are two different cost functions whose optimal distributions do not coincide. In particular, a single-frequency HTP might yield acceptable HTQ, but poor target coverage. This effect is apparent in figures 8 and 9: even when the HTQ closely fluctuates around 1 , the $\mathrm{TC}_{x}$ exhibits a strong frequency-dependent trend, quickly falling below the acceptance threshold. To satisfy both $\mathrm{HTQ}<1$ and $\mathrm{TC}_{x}>75 \%$, one must carefully select the optimal operating frequency, which depends on the applicator in use, the patient's anatomy, and the target shape. For the larynx case used in this study, the optimal frequency lies around $400 \mathrm{MHz}$, while for the meninges $500 \mathrm{MHz}$ appears to be a better choice. This motivates the need for wide-band applicators and for treatment planning tools capable of seeking the best treatment frequency within the available band. Figures 8 and 9 further show that in a multi-frequency setting, the requirements on the HTQ and $\mathrm{TC}_{x}$ can be more easily fulfilled when two frequencies are selected simultaneously. In other words, the multi-frequency approach helps reaching the multiple objectives needed for a successful hyperthermia dose delivery.

The proposed i-TR technique aims at quickly determining such an overall optimal SAR distribution by exploiting the different frequency components to minimize a custom cost function, the HCQ. This cost function is designed to reach the multiple objectives above while enabling a TR-based iterative approach. The SAR-based results, section 4.1, suggest that the method is successful in finding viable solutions for a given problem, independently of the target's size, location, morphology or composition. They further indicate that the method is robust to problems involving mixed polarization axes. The quality of the $\mathrm{i}$-TR solution can depend on the selected set of operating frequencies, however the high execution speed makes it possible to evaluate all frequency combinations and always determine one viable solution. Note that this solution might not be the global optimum in terms of HCQ. We further point out that, although there is no guarantee of improvement of 
an initial TR solution by $\mathrm{i}-\mathrm{TR}$, the process is stable as it can never return a solution worse than the initial one in terms of HCQ.

The thermal validation, section 4.4, fundamentally confirms the i-TR's ability to provide viable HTP solutions and further emphasizes the difference in $\mathrm{HTQ}$ and $\mathrm{TC}_{x}$ as treatment planning goals. In the larynx case, for instance, all HTP algorithms exhibit similar HTQ values below 1 at the selected frequency combination. However, only for i-TR and PS this translates into high thermal doses, thanks to their higher SAR coverage. EV and classic TR fail to fully exploit the applicator's heating capabilities, falling short of $2{ }^{\circ} \mathrm{C}$ on average in $T_{50}$ for the two cases. The small improvement in HTQ brought about by PS, negligible at a first glance, results in a significantly higher median tumor temperature. It is however i-TR to perform best in the larynx, with half a degree higher $T_{50}$ than PS. This is likely due to the compromise that HCQ seeks between low HTQ and high coverage. In the meninges, the situation is similar for EV and TR, while i-TR provides again a solution very close to PS, with only half a degree lower $T_{50}$. While it is not possible to draw general conclusions about the relationship between $\mathrm{HTQ}, \mathrm{TC}_{x}, \mathrm{HCQ}$ and the resulting temperature distribution due to the limited amount of test cases, the thermal validation indicates that the HTP solutions provided by i-TR can be considered as clinically relevant.

Regarding EV, many improvements have been suggested over the years in an effort to exploit this extremely fast solution method. Works such as those of Köhler et al (2001) and Mestrom et al (2014) apply weighing functions to the SAR distribution in order to reduce the SAR peak in the healthy tissues and improve the SAR homogeneity within the target. The weight distribution itself, however, needs to be determined by means of an iterative procedure. Moreover, these modifications only apply to the single-frequency scenario. In a multifrequency setting, the fundamental single-frequency limitation of the EV cost function $\left(\overline{\mathrm{SAR}}_{\mathrm{T}} / \overline{\mathrm{SAR}}_{\mathrm{R}}\right)$ needs to be resolved to fully exploit the treatment capabilities of wide-band applicators (Zanoli and Trefná 2020). Recently, an emerging time- and frequency-multiplexed beam-forming method has been proposed by Kuehne et al (2020). The method is based on quadratic programming (QP) and is therefore extremely fast in determining the optimal set of frequencies, steering parameters and temporal sequences that best approximate a specified SAR pattern. However, the method does not determine the optimal SAR pattern in itself. While this is a very powerful approach, we believe that a relevant comparison between applicators should be grounded on clinically relevant HTP quality indicators, such as $\mathrm{HTQ}$ and $\mathrm{TC}_{x}$. These have been shown to correlate with high tumor temperatures (Bellizzi et al 2019), which are the ultimate goal in hypethermia. However, since these indicators involve a nonlinear step, they cannot be solved for with fast QP-based algorithms.

The proposed i-TR technique runs in nearly real-time even in the case of high-resolution model matrices such as those considered in this study. The $2 \mathrm{~mm}$ resolution is necessary to model the thin anatomical features of the vertebrae and the skull, which strongly affect the wave propagation. Current clinical HTP tools for online complaint-adaptive steering use coarse resolutions of $5 \mathrm{~mm}$ for $70 \mathrm{MHz}$ and $434 \mathrm{MHz}$ applicators to be able to quickly recompute the steering parameters (Rijnen et al 2013, Kok et al 2017). For UWB applicators utilizing frequencies up to $800 \mathrm{MHz}$, this resolution is no longer sufficient, especially when considering tumors in the brain.

We point out that the proposed i-TR technique differs substantially from the iterative TR method developed by Montaldo and colleagues for ultrasound applications (Montaldo et al 2004). In HIFU, the aim is to restore the sharp TR focal spot after distortions caused by lossy and heterogeneous media. In MW-HT, the problem faced is the opposite: to de-focus the sharp SAR peak caused by TR in order to achieve better coverage across the whole target volume. As a final consideration, we note that the i-TR implementation described in this paper is not unique. For example, the cold-spot $\rightarrow H S$ order in the iterative scheme, figure 3, could be swapped. Similarly, the identification of hot- and cold-spots could be performed right before each spot iteration, as done here, or only once before both iterations. During internal tests, we could not identify any major effects of these choices on the HTP outcome.

\section{Conclusion}

The proposed i-TR beam-forming technique is shown to deliver HTP solutions equivalent to those provided by global optimizers such as PS, while being orders of magnitude faster. Results indicate that the method is robust to different array and target configurations. The custom HCQ cost function solved for by the iterative method leads to solutions that exhibit a good compromise between raw power deposition in the tumor, HS suppression in healthy tissues, and target coverage even in deeper regions. Overall, the described i-TR technique provides the means for fast comparisons of a large number of potential array configurations and frequency combinations to optimally treat a given patient. In a clinical setting, i-TR might be further extended for use as a real-time steering parameter re-optimization procedure upon localized complaint from the patient. 


\section{Acknowledgments}

This work was financially supported by the VINN EXcellence Center of ChaseOn (Chalmers Antenna Systems) and The Swedish Childhood Cancer Fund.

\section{ORCID iDs}

Massimiliano Zanoli (ii) https://orcid.org/0000-0002-3149-6905

Hana Dobšiček Trefná iㅣ https://orcid.org/0000-0001-6025-0819

\section{References}

Bardati F, Borrani A, Gerardino A and Lovisolo G A 1995 SAR optimization in a phased array radiofrequency hyperthermia system IEEE Trans. Biomed. Eng. 42 1201-7

Battaglia G, Morabito A, Palmeri R and Isernia T 2020 Constrained focusing of vector fields intensity in near zone and/or complex scenarios as a low-dimensional global optimization J. Electromagn. Waves Appl. 34 1977-89

Bellizzi G G, Crocco L, Battaglia G M and Isernia T 2017 Multi-frequency constrained SAR focusing for patient specific hyperthermia treatment IEEE J. Electromagn. RF Microwaves Med. Biol. 174-80

Bellizzi G G, Drizdal T, van Rhoon G C, Crocco L, Isernia T and Paulides M M 2019 Predictive value of sar based quality indicators for head and neck hyperthermia treatment quality Int. J. Hyperth. 36 455-64

Böhm M, Kremer J and Louis A 1993 Efficient algorithm for computing optimal control of antennas in hyperthermia Surv. Math. Ind. 3 233-51

Canters R A, Paulides M M, Franckena M F, van der Zee J and van Rhoon G C 2012 Implementation of treatment planning in the routine clinical procedure of regional hyperthermia treatment of cervical cancer: an overview and the Rotterdam experience Int. J. Hyperth. 28 $570-81$

Canters R, Franckena M, van der Zee J and Van Rhoon G 2010 Optimizing deep hyperthermia treatments: are locations of patient pain complaints correlated with modelled SAR peak locations? Phys. Med. Biol. 56 439-51

Canters R, Paulides M, Franckena M, Mens J and Van Rhoon G 2013 Benefit of replacing the sigma-60 by the sigma-eye applicator Strahlentherapie und Onkologie 189 74-80

Canters R, Wust P, Bakker J and Van Rhoon G 2009 A literature survey on indicators for characterisation and optimisation of SAR distributions in deep hyperthermia, a plea for standardisation Int. J. Hyperth. 25 593-608

Cihoric N et al 2015 Hyperthermia-related clinical trials on cancer treatment within the ClinicalTrials.gov registry Int. J. Hyperth. 31 609-14

Converse M, Bond E J, Veen B and Hagness C 2006 A computational study of ultra-wideband versus narrowband microwave hyperthermia for breast cancer treatment IEEE Trans. Microwave Theory Tech. 542169-80

Dassault Systèmes SE, Vélizy-Villacoublay, France 2019 CST Studio Suite 2019

Datta N, Ordóñez S G, Gaipl U, Paulides M, Crezee H, Gellermann J, Marder D, Puric E and Bodis S 2015 Local hyperthermia combined with radiotherapy and-/or chemotherapy: recent advances and promises for the future Cancer Treat. Rev. 41 742-53

Datta N R, Rogers S, Ordóñez S G, Puric E and Bodis S 2016 Hyperthermia and radiotherapy in the management of head and neck cancers: a systematic review and meta-analysis Int. J. Hyperth. 32 31-40

De Greef M, Kok H, Correia D, Bel A and Crezee J 2010 Optimization in hyperthermia treatment planning: the impact of tissue perfusion uncertainty Med. Phys. 37 4540-50

Elming P B, Sørensen B S, Oei A L, Franken N A, Crezee J, Overgaard J and Horsman M R 2019 Hyperthermia: the optimal treatment to overcome radiation resistant hypoxia Cancers 1160

Fatehi D, Van Der Zee J, Notenboom A and van Rhoon G C 2007 Comparison of intratumor and intraluminal temperatures during locoregional deep hyperthermia of pelvic tumors Strahlentherapie und Onkologie 183 479-86

Fink M, Montaldo G and Tanter M 2004 Time reversal acoustics IEEE Ultrasonics Symp., 2004, vol 2 (Piscataway, NJ: IEEE) pp 850-9

Gavazzi S, van Lier A L, Zachiu C, Jansen E, Lagendijk J J W, Stalpers L J A, Crezee H and Kok H P 2020 Advanced patient-specific hyperthermia treatment planning Int. J. Hyperth. 37 992-1007

Gosselin M-C et al 2014 Development of a new generation of high-resolution anatomical models for medical device evaluation: the Virtual Population 3.0 Phys. Med. Biol. $595287-303$

Guérin B, Villena J F, Polimeridis A G, Adalsteinsson E, Daniel L, White J K, Rosen B R and Wald L L 2018 Computation of ultimate SAR amplification factors for radiofrequency hyperthermia in non-uniform body models: impact of frequency and tumour location Int. J. Hyperth. $3487-100$

Guo B, Xu L and Li J 2005 Time reversal based microwave hyperthermia treatment of breast cancer Microwave Opt. Technol. Lett. 47 335-8

Hasgall P, Neufeld E, Gosselin M, Klingenböck A, Kuster N, Hasgall P and Gosselin M 2012 IT’IS database for thermal and electromagnetic parameters of biological tissues Version 4.0 (15 May 2018) (https://doi.org/10.13099/VIP21000-04-0. itis.swiss/database)

Iero D A, Crocco L, Isernia T and Korkmaz E 2016 Optimal focused electromagnetic hyperthermia treatment of breast cancer 201610 th European Conf. on Antennas and Propagation (EuCAP) (Piscataway, NJ: IEEE) pp 1-2

Köhler T, Maass P, Wust P and Seebass M 2001 A fast algorithm to find optimal controls of multiantenna applicators in regional hyperthermia Phys. Med. Biol. 46 2503-14

Kok H P, Gellermann J, van den Berg C A, Stauffer P R, Hand J W and Crezee J 2013 Thermal modelling using discrete vasculature for thermal therapy: a review Int. J. Hyperth. 29336-45

Kok H P, Korshuize-van Straten L, Bakker A, de Kroon-Oldenhof R, Geijsen E D, Stalpers L J and Crezee J 2017 Online adaptive hyperthermia treatment planning during locoregional heating to suppress treatment-limiting hot spots Int. J. Radiat. Onco. Biol.Phys. 99 1039-47

Kok H, Wust P, Stauffer P R, Bardati F, Van Rhoon G and Crezee J 2015 Current state of the art of regional hyperthermia treatment planning: a review Radiat. Oncol. 10196

Kuehne A, Oberacker E, Waiczies H and Niendorf T 2020 Solving the time-and frequency-multiplexed problem of constrained radiofrequency induced hyperthermia Cancers 121072 
Lang J, Erdmann B and Seebass M 1999 Impact of nonlinear heat transfer on temperature control in regional hyperthermia IEEE Trans. Biomed. Eng. $461129-38$

Leduc N, Okita K, Sugiyama K, Takagi S and Matsumoto Y 2012 Focus control in HIFU therapy assisted by time-reversal simulation with an iterative procedure for hot spot elimination J. Biomech. Sci. Eng. $743-56$

Lee H K, Antell A G, Perez C A, Straube W L, Ramachandran G, Myerson R J, Emami B, Molmenti E P, Buckner A and Lockett M A 1998 Superficial hyperthermia and irradiation for recurrent breast carcinoma of the chest wall: prognostic factors in 196 tumors Int. J. Radiat. Oncol. Biol. Phys. 40 365-75

Lerosey G, De Rosny J, Tourin A, Derode A, Montaldo G and Fink M 2004 Time reversal of electromagnetic waves Phys. Rev. Lett. 92 193904

Mestrom R, Van Engelen J, Van Beurden M, Paulides M, Numan W and Tijhuis A 2014 A refined eigenvalue-based optimization technique for hyperthermia treatment planning The 8th European Conf. on Antennas and Propagation (EuCAP 2014) (Piscataway, NJ: IEEE) pp 2010-3

Montaldo G, Tanter M and Fink M 2004 Real time inverse filter focusing through iterative time reversal J. Acoust. Soc. Am. $115768-75$

Myerson R J, Perez C A, Emami B, Straube W, Kuske R R, Leybovich L and Von Gerichten D 1990 Tumor control in long-term survivors following superficial hyperthermia Int. J. Radiat. Oncol. Biol. Phys. $181123-9$

Paulides M, Bakker J, Linthorst M, Van der Zee J, Rijnen Z, Neufeld E, Pattynama P, Jansen P, Levendag P and Van Rhoon G 2010 The clinical feasibility of deep hyperthermia treatment in the head and neck: new challenges for positioning and temperature measurement Phys. Med. Biol. 55 2465-80

Paulides M, Bakker J, Neufeld E, Zee J V D, Jansen P, Levendag P and Van Rhoon G 2007 The HYPERcollar: a novel applicator for hyperthermia in the head and neck Int. J. Hyperth. 23 567-76

Paulides M M, Stauffer P R, Neufeld E, Maccarini P F, Kyriakou A, Canters R A, Diederich C J, Bakker J F and Van Rhoon G C 2013 Simulation techniques in hyperthermia treatment planning Int. J. Hyperth. 29346-57

Paulides M M, Verduijn G M and Van Holthe N 2016 Status quo and directions in deep head and neck hyperthermia Radiat. Oncol. 1121

Paulides M, Trefna H D, Curto S and Rodrigues D 2020 Recent technological advancements in radiofrequency-and microwave-mediated hyperthermia for enhancing drug delivery Adv. Drug Deliv. Rev. 163-164 3-18

Piotrowski A P, Napiorkowski J J and Piotrowska A E 2020 Population size in particle swarm optimization Swarm Evolutionary Comput. 58 100718

Qi N, Zhang M, Wittig T and Prokop A 2008 Application of CST time domain algorithm in the electromagnetic simulation standard of the SAR for mobile phone 2008 Int. Conf. on Microwave and Millimeter Wave Technology vol 4 (Piscataway, NJ: IEEE) pp 1717-20

Rijnen Z, Bakker J F, Canters R A, Togni P, Verduijn G M, Levendag P C, Van Rhoon G C and Paulides M M 2013 Clinical integration of software tool vedo for adaptive and quantitative application of phased array hyperthermia in the head and neck Int. J. Hyperth. 29 181-93

Schooneveldt G, Trefná H D, Persson M, De Reijke T M, Blomgren K, Kok H P and Crezee H 2019 Hyperthermia treatment planning including convective flow in cerebrospinal fluid for brain tumour hyperthermia treatment using a novel dedicated paediatric brain applicator Cancers 111183

Song C W 1984 Effect of local hyperthermia on blood flow and microenvironment: a review Cancer Res. 44 4721s-30s

Takook P, Persson M, Gellermann J and Trefná H D 2017 Compact self-grounded bow-tie antenna design for an UWB phased-array hyperthermia applicator Int. J. Hyperth. 33 387-400

Takook P, Persson M and Trefná H D 2018 Performance evaluation of hyperthermia applicators to heat deep-seated brain tumors IEEE J. Electromagn. RF Microwaves Med. Biol. 2 18-24

Takook P, Trefná H D, Fhager A and Persson M 2015 Evaluation of the 3D time reversal method for hyperthermia treatment planning in head and neck tumors 2015 9th European Conf. on Antennas and Propagation (EuCAP) (Piscataway, NJ: IEEE) pp 1-5

Takook P, Trefná H D, Zeng X, Fhager A and Persson M 2017 A computational study using time reversal focusing for hyperthermia treatment planning Prog. Electromagn. Res. 73 117-30

The MathWorks Inc. 2019 MATLAB R2019 (Natick, MA: The MathWorks Inc.)

Thomas J-L and Fink M A 1996 Ultrasonic beam focusing through tissue inhomogeneities with a time reversal mirror: application to transskull therapy IEEE Trans. Ultrason. Ferroelectr. Freq. Control $431122-9$

Togni P, Rijnen Z, Numan W, Verhaart R, Bakker J, Van Rhoon G and Paulides M 2013 Electromagnetic redesign of the HYPERcollar applicator: toward improved deep local head-and-neck hyperthermia Phys. Med. Biol. 58 5997-6009

Trefná H D, Martinsson B, Petersson T, Renström N, Torstensson M, Ravanis J, Kok P and Persson M 2017 Multifrequency approach in hyperthermia treatment planning: impact of frequency on SAR distribution in head and neck 2017 11th European Conf. on Antennas and Propagation (EUCAP) (Piscataway, NJ: IEEE) pp 3710-2

Trefná H D, Togni P, Shiee R, Vrba J and Persson M 2012 Design of a wideband multi-channel system for time reversal hyperthermia Int. J. Hyperth. 28 175-83

Trefná H D, Vrba J and Persson M 2010a Evaluation of a patch antenna applicator for time reversal hyperthemia Int. J. Hyperth. 26 185-97

Trefná H D, Vrba J and Persson M 2010b Time-reversal focusing in microwave hyperthermia for deep-seated tumors Phys. Med. Biol. 55 2167-85

Winter L, Oezerdem C, Hoffmann W, van de Lindt T, Periquito J, Ji Y, Ghadjar P, Budach V, Wust P and NiendorfT 2015 Thermal magnetic resonance: physics considerations and electromagnetic field simulations up to 23.5 Tesla (1 ghz) Radiat. Oncol. $101-12$

Wust P, Fähling H, Helzel T, Kniephoff M, Wlodarczyk W, Mönich G and Felix R 1998 Design and test of a new multi-amplifier system with phase and amplitude control Int. J. Hyperth. 14459-77

Yarmolenko P S, Moon E J, Landon C, Manzoor A, Hochman D W, Viglianti B L and Dewhirst M W 2011 Thresholds for thermal damage to normal tissues: an update Int. J. Hyperth. 27 320-43

Zanoli M and Trefná H D 2020 Suitability of eigenvalue beam forming for discrete multi-frequency hyperthermia treatment planning Med. Phys. submitted 